TRANSACTIONS OF THE

AMERICAN MATHEMATICAL SOCIETY

Volume 363, Number 11, November 2011, Pages 6111-6141

S 0002-9947(2011)05371-7

Article electronically published on April 28, 2011

\title{
ROOT POLYTOPES, TRIANGULATIONS, AND THE SUBDIVISION ALGEBRA, II
}

\author{
KAROLA MÉSZÁROS
}

\begin{abstract}
The type $C_{n}$ root polytope $\mathcal{P}\left(C_{n}^{+}\right)$is the convex hull in $\mathbb{R}^{n}$ of the origin and the points $e_{i}-e_{j}, e_{i}+e_{j}, 2 e_{k}$ for $1 \leq i<j \leq n, k \in[n]$. Given a graph $G$, with edges labeled positive or negative, associate to each edge $e$ of $G$ a vector $\mathrm{v}(e)$ which is $e_{i}-e_{j}$ if $e=(i, j), i<j$, is labeled negative and $e_{i}+e_{j}$ if it is labeled positive. For such a signed graph $G$, the associated root polytope $\mathcal{P}(G)$ is the intersection of $\mathcal{P}\left(C_{n}^{+}\right)$with the cone generated by the vectors $\mathrm{v}(e)$, for edges $e$ in $G$. The reduced forms of a certain monomial $m[G]$ in commuting variables $x_{i j}, y_{i j}, z_{k}$ under reductions derived from the relations of a bracket algebra of type $C_{n}$, can be interpreted as triangulations of $\mathcal{P}(G)$. Using these triangulations, the volume of $\mathcal{P}(G)$ can be calculated. If we allow variables to commute only when all their indices are distinct, then we prove that the reduced form of $m[G]$, for "good" graphs $G$, is unique and yields a canonical triangulation of $\mathcal{P}(G)$ in which each simplex corresponds to a noncrossing alternating graph in a type $C$ sense. A special case of our results proves a conjecture of A. N. Kirillov about the uniqueness of the reduced form of a Coxeter type element in the bracket algebra of type $C_{n}$. We also study the bracket algebra of type $D_{n}$ and show that a family of monomials has unique reduced forms in it. A special case of our results proves a conjecture of A. N. Kirillov about the uniqueness of the reduced form of a Coxeter type element in the bracket algebra of type $D_{n}$.
\end{abstract}

\section{INTRODUCTION}

In this paper we develop the connection between triangulations of type $C_{n}$ root polytopes and a commutative algebra $\mathcal{S}\left(C_{n}\right)$, the subdivision algebra of type $C_{n}$ root polytopes. A type $C_{n}$ root polytope is a convex hull of the origin and some of the points $e_{i}-e_{j}, e_{i}+e_{j}, 2 e_{k}$ for $1 \leq i<j \leq n, k \in[n]$, where $e_{i}$ denotes the $i^{\text {th }}$ standard basis vector in $\mathbb{R}^{n}$. A polytope $\mathcal{P}(m)$ corresponds to each monomial $m \in \mathcal{S}\left(C_{n}\right)$, and each relation of the algebra equating a monomial with three others, $m_{0}=m_{1}+m_{2}+m_{3}$, can be interpreted as cutting the polytope $\mathcal{P}\left(m_{0}\right)$ into two polytopes $\mathcal{P}\left(m_{1}\right)$ and $\mathcal{P}\left(m_{2}\right)$ with interiors disjoint such that $\mathcal{P}\left(m_{1}\right) \cap \mathcal{P}\left(m_{2}\right)=$ $\mathcal{P}\left(m_{3}\right)$, thus the name subdivision algebra for $\mathcal{S}\left(C_{n}\right)$.

Received by the editors October 6, 2009 and, in revised form April 17, 2010.

2010 Mathematics Subject Classification. Primary 05E15, 16S99, 51M25, 52B11.

Key words and phrases. Root polytope, type $C_{n}$, type $D_{n}$, triangulation, volume, Ehrhart polynomial, noncrossing alternating graph, subdivision algebra, bracket algebra, reduced form, noncommutative Gröbner basis.

(C)2011 American Mathematical Society Reverts to public domain 28 years from publication 
A subdivision algebra $\mathcal{S}\left(A_{n}\right)$ for type $A_{n}$ root polytopes was studied in [M], yielding an exciting interplay between polytopes and algebras. The algebra $\mathcal{S}\left(A_{n}\right)$ is related to the algebras studied by Fomin and Kirillov in [FK] and by Kirillov in [K1, which have tight connections to Schubert calculus. Using techniques for polytopes, the algebra $\mathcal{S}\left(A_{n}\right)$ can be better understood, and using the properties of $\mathcal{S}\left(A_{n}\right)$, results for root polytopes can be deduced. The subdivision algebra $\mathcal{S}\left(C_{n}\right)$ is a type $C_{n}$ generalization of $\mathcal{S}\left(A_{n}\right)$, and its intimate connection to type $C_{n}$ root polytopes is displayed by a variety of results obtained by using this connection.

Root polytopes were first defined by Postnikov in [P], although the full root polytope of type $A_{n}$ already appeared in the work of Gelfand, Graev and Postnikov GGP], where they gave a canonical triangulation of it into simplices corresponding to noncrossing alternating trees. Properties of this triangulation are studied in $\mathbf{S} 2$, Exercise 6.31]. Canonical triangulations for a family of type $A_{n}$ root polytopes were constructured in [M] extending the result of [GGP. In this paper we define type $C_{n}$ analogs for noncrossing and alternating graphs, and show that a family of type $C_{n}$ root polytopes, containing the full root polytope, has canonical triangulations into simplices corresponding to noncrossing alternating graphs. Using the canonical triangulations we compute the volumes for these root polytopes.

The subdivision algebra $\mathcal{S}\left(C_{n}\right)$ is closely related to the noncommutative bracket algebra $\mathcal{B}^{n c}\left(C_{n}\right)$ of type $C_{n}$ defined by A. N. Kirillov $\mathrm{K} 2$. Kirillov conjectured the uniqueness of the reduced form of a Coxeter type element in $\mathcal{B}^{n c}\left(C_{n}\right)$. As the algebras $\mathcal{S}\left(C_{n}\right)$ and $\mathcal{B}^{n c}\left(C_{n}\right)$ have over ten not-so-simple-looking relations, we postpone their definitions and the precise statement of Kirillov's conjecture till Section 2 While at first sight the relations of $\mathcal{B}^{n c}\left(C_{n}\right)$ might appear rather mysterious, we interpret them similarly to the relations of $\mathcal{S}\left(C_{n}\right)$, as certain subdivisions of root polytopes. This connection ultimately yields a proof of Kirillov's conjecture along with more general theorems on reduced forms, of which there are two types. In the noncommutative algebra $\mathcal{B}^{n c}\left(C_{n}\right)$ we show that for a family of monomials $\mathcal{M}$, including the Coxeter type element defined by Kirillov, the reduced form is unique. In the commutative algebra $\mathcal{S}\left(C_{n}\right)$ and the commutative counterpart $\mathcal{B}^{c}\left(C_{n}\right)=\mathcal{B}^{n c}\left(C_{n}\right) /\left[\mathcal{B}^{n c}\left(C_{n}\right), \mathcal{B}^{n c}\left(C_{n}\right)\right]$ of $\mathcal{B}^{n c}\left(C_{n}\right)$, the reduced forms are not unique; however, we show that the number of monomials in a reduced form of $m \in \mathcal{M}$ is independent of the order of the reductions performed.

We also study the noncommutative bracket algebra $\mathcal{B}\left(D_{n}\right)$ of type $D_{n}$ defined by A. N. Kirillov K2. Using noncommutative Gröbner bases techniques we prove that a family of monomials has unique reduced forms in it. A special case of our results proves a conjecture of A. N. Kirillov about the uniqueness of the reduced form of a Coxeter type element in the bracket algebra of type $D_{n}$.

This paper is organized as follows. In Section 2 we give the definition of $\mathcal{B}^{n c}\left(C_{n}\right)$, as well as two related commutative algebras $\mathcal{B}^{c}\left(C_{n}\right)$ and $\mathcal{S}\left(C_{n}\right)$. We also state Kirillov's conjecture pertaining to $\mathcal{B}^{n c}\left(C_{n}\right)$ in Section 2 , In Section 3 we introduce signed graphs, define the type $C$ analogue of alternating graphs, and show how to reformulate the relations of the algebras $\mathcal{B}^{c}\left(C_{n}\right), \mathcal{S}\left(C_{n}\right)$ into reductions on graphs. In Section 4 we introduce coned root polytopes of type $C_{n}$ and state the Reduction Lemma which connects root polytopes and the algebras $\mathcal{B}^{n c}\left(C_{n}\right), \mathcal{B}^{c}\left(C_{n}\right), \mathcal{S}\left(C_{n}\right)$. In Section 5 we prove a characterization of the vertices of coned type $C_{n}$ root polytopes, while in Section 6 we prove the Reduction Lemma. In Section 7 we establish the relation between volumes of root polytopes and reduced forms of monomials 
in the algebras $\mathcal{B}^{c}\left(C_{n}\right), \mathcal{S}\left(C_{n}\right)$ using the Reduction Lemma. In Section 8 we reformulate the noncommutative relations of $\mathcal{B}^{n c}\left(C_{n}\right)$ in terms of edge-labeled graphs and define well-structured and well-labeled graphs, key for our further considerations. In Section 9 we prove a simplified version of Kirillov's conjecture, construct a canonical triangulation for the full type $C_{n}$ root polytope $\mathcal{P}\left(C_{n}^{+}\right)$and calculate its volume. In Section 10 we generalize Kirillov's conjecture to all monomials arising from well-structured and well-labeled graphs and give the triangulations and volumes of the corresponding root polytopes. In Section 11 we prove the general form of Kirillov's conjecture in a weighted bracket algebra $\mathcal{B}^{\beta}\left(C_{n}\right)$ and show a way to calculate Ehrhart polynomials of certain type $C_{n}$ root polytopes. In Section 12 the definition of $\mathcal{B}\left(D_{n}\right)$ is given along with Kirillov's conjecture pertaining to it. In Section 13 combinatorial results regarding a family of monomials are proved. Finally, in Section 14 we prove a general result on the reduced forms of monomials implying Kirillov's type $D_{n}$ conjecture.

\section{The BRACKet AND SUbDivision ALgebras of TyPe $C_{n}$}

In this section the definition of the bracket algebra $\mathcal{B}^{n c}\left(C_{n}\right)$ is given, along with a conjecture of Kirillov pertaining to it. We introduce the subdivision algebra $\mathcal{S}\left(C_{n}\right)$, which, as its name suggests, will be shown to govern subdivisions of type $C_{n}$ root polytopes.

Kirillov [K2] defined the algebra that we are denoting by $\mathcal{B}^{n c}\left(C_{n}\right)$ as a type $B_{n}$ bracket algebra $\mathcal{B}\left(B_{n}\right)$, but since we can interpret its generating variables as corresponding to either the type $B_{n}$ and type $C_{n}$ roots, we refer to it as a type $C_{n}$ bracket algebra $\mathcal{B}^{n c}\left(C_{n}\right)$. The reason for our desire to designate $\mathcal{B}^{n c}\left(C_{n}\right)$ as a type $C_{n}$ algebra is its essential link to type $C_{n}$ root polytopes, which we develop in this paper. Here we define a simplified form of the bracket algebra $\mathcal{B}^{n c}\left(C_{n}\right)$; for a more general definition, see Section 11 .

Let the bracket algebra $\mathcal{B}^{n c}\left(C_{n}\right)$ of type $C_{n}$ be an associative algebra over $\mathbb{Q}$ with a set of generators $\left\{x_{i j}, y_{i j}, z_{i} \mid 1 \leq i \neq j \leq n\right\}$ subject to the following relations:

(1) $x_{i j}+x_{j i}=0, y_{i j}=y_{j i}$, for $i \neq j$,

(2) $z_{i} z_{j}=z_{j} z_{i}$

(3) $x_{i j} x_{k l}=x_{k l} x_{i j}, y_{i j} x_{k l}=x_{k l} y_{i j}, y_{i j} y_{k l}=y_{k l} y_{i j}$, for $i<j, k<l$ distinct,

(4) $z_{i} x_{k l}=x_{k l} z_{i}, z_{i} y_{k l}=y_{k l} z_{i}$, for all $i \neq k, l$,

(5) $x_{i j} x_{j k}=x_{i k} x_{i j}+x_{j k} x_{i k}$, for $1 \leq i<j<k \leq n$,

(5') $x_{j k} x_{i j}=x_{i j} x_{i k}+x_{i k} x_{j k}$, for $1 \leq i<j<k \leq n$,

(6) $x_{i j} y_{j k}=y_{i k} x_{i j}+y_{j k} y_{i k}$, for $1 \leq i<j<k \leq n$,

(6') $y_{j k} x_{i j}=x_{i j} y_{i k}+y_{i k} y_{j k}$, for $1 \leq i<j<k \leq n$,

(7) $x_{i k} y_{j k}=y_{j k} y_{i j}+y_{i j} x_{i k}$, for $1 \leq i<j<k \leq n$,

(7') $y_{j k} x_{i k}=y_{i j} y_{j k}+x_{i k} y_{i j}$, for $1 \leq i<j<k \leq n$,

(8) $y_{i k} x_{j k}=x_{j k} y_{i j}+y_{i j} y_{i k}$, for $1 \leq i<j<k \leq n$,

(8') $x_{j k} y_{i k}=y_{i j} x_{j k}+y_{i k} y_{i j}$, for $1 \leq i<j<k \leq n$,

(9) $x_{i j} z_{j}=z_{i} x_{i j}+y_{i j} z_{i}+z_{j} y_{i j}$, for $i<j$,

$\left(9^{\prime}\right) z_{j} x_{i j}=x_{i j} z_{i}+z_{i} y_{i j}+y_{i j} z_{j}$, for $i<j$.

Let $w_{C_{n}}=\prod_{i=1}^{n-1} x_{i, i+1} z_{n}$ be a Coxeter type element in $\mathcal{B}^{n c}\left(C_{n}\right)$ and let $P_{n}^{\mathcal{B}}$ be the polynomial in the variables $x_{i j}, y_{i j}, z_{i}, 1 \leq i \neq j \leq n$ obtained from $w_{C_{n}}$ by successively applying the defining relations $(1)-\left(9^{\prime}\right)$ in any order until unable to 
do so. We call $P_{n}^{\mathcal{B}}$ a reduced form of $w_{C_{n}}$ and consider the process of successively applying the defining relations $(5)-\left(9^{\prime}\right)$ as a reduction process, with possible commutations (2)-(4) between reductions, as we show in the following example:

$$
\begin{aligned}
\mathbf{x}_{\mathbf{1 2}} \mathbf{x}_{\mathbf{2 3}} z_{3} \rightarrow & x_{13} \underline{x_{12} z_{3}}+x_{23} \mathbf{x}_{\mathbf{1 3}} \mathbf{z}_{\mathbf{3}} \\
\rightarrow & \mathbf{x}_{\mathbf{1 3}} \mathbf{z}_{\mathbf{3}} x_{12}+x_{23} z_{1} x_{13}+\mathbf{x}_{\mathbf{2 3}} \mathbf{y}_{13} z_{1}+\mathbf{x}_{\mathbf{2 3}} \mathbf{z}_{\mathbf{3}} y_{13} \\
\rightarrow & z_{1} x_{13} x_{12}+y_{13} z_{1} x_{12}+z_{3} y_{13} x_{12}+x_{23} z_{1} x_{13}+y_{12} x_{23} z_{1}+y_{13} y_{12} z_{1} \\
& +z_{2} \mathbf{x}_{\mathbf{2 3}} \mathbf{y}_{13}+y_{23} z_{2} y_{13}+z_{3} y_{23} y_{13} \\
\rightarrow & z_{1} x_{13} x_{12}+y_{13} z_{1} x_{12}+z_{3} y_{13} x_{12}+x_{23} z_{1} x_{13}+y_{12} x_{23} z_{1} \\
& +y_{13} y_{12} z_{1}+z_{2} y_{12} x_{23}+z_{2} y_{13} y_{12}+y_{23} z_{2} y_{13}+z_{3} y_{23} y_{13} .
\end{aligned}
$$

In the example above the pair of variables on which one of reductions $(5)-\left(9^{\prime}\right)$ is performed is in boldface, and the variables which we commute according to one of (2)-(4) are underlined.

Conjecture 1 (Kirillov [K2]). Apart from applying the relations (1)-(4), the reduced form $P_{n}^{\mathcal{B}}$ of $w_{C_{n}}$ does not depend on the order in which the reductions are performed.

Note that the above statement does not hold true for any monomial. We show one simple example of how it fails:

$$
\begin{aligned}
& \boldsymbol{x}_{\mathbf{1 2}} \boldsymbol{x}_{\mathbf{2 3}} y_{13} \rightarrow x_{13} x_{12} y_{13}+x_{23} x_{13} y_{13}, \\
& x_{12} \boldsymbol{x}_{\mathbf{2 3}} \boldsymbol{y}_{\mathbf{1 3}} \rightarrow x_{12} y_{12} x_{23}+x_{12} y_{13} y_{12} .
\end{aligned}
$$

Note that we reduced the monomial $x_{12} x_{23} y_{23}$ in two different ways yielding two different polynomials. The reader can also check another example of this phenomenon by reducing the monomial $y_{14} x_{24} y_{34}$ in two different ways to obtain two different reduced forms.

We prove Conjecture 1 in Section 9, as well as its generalizations in Sections 10 and 11 We first define and study a commutative algebra $\mathcal{S}\left(C_{n}\right)$ closely related to $\mathcal{B}^{n c}\left(C_{n}\right)$, though more complicated than its commutative counterpart, $\mathcal{B}^{c}\left(C_{n}\right)=\mathcal{B}^{n c}\left(C_{n}\right) /\left[\mathcal{B}^{n c}\left(C_{n}\right), \mathcal{B}^{n c}\left(C_{n}\right)\right]$, which is simply the commutative associative algebra over $\mathbb{Q}$ with a set of generators $\left\{x_{i j}, y_{i j}, z_{i} \mid 1 \leq i \neq j \leq n\right\}$ subject to relations (1) and (5)-( $\left.9^{\prime}\right)$ from above. Our motivation for defining $\mathcal{S}\left(C_{n}\right)$ is a natural correspondence between the relations of $\mathcal{S}\left(C_{n}\right)$ and ways to subdivide type $C_{n}$ root polytopes, which correspondence is made precise in the Reduction Lemma (Lemma 3). In order to emphasize this connection, we call $\mathcal{S}\left(C_{n}\right)$ the subdivision algebra of type $C_{n}$. The subalgebra $\mathcal{S}\left(A_{n-1}\right)$ of $\mathcal{S}\left(C_{n}\right)$ generated by $\left\{x_{i j} \mid 1 \leq i \neq j \leq n\right\}$ has been studied in [M], and an analogous correspondence between the relations of $\mathcal{S}\left(A_{n-1}\right)$ and ways to subdivide type $A_{n-1}$ root polytopes has been established. Moreover, results in the spirit of Conjecture 1 for type $A_{n-1}$ can also be found in $[\mathrm{M}]$.

Let the subdivision algebra $\mathcal{S}\left(C_{n}\right)$ be the commutative algebra over $\mathbb{Q}[\beta]$, where $\beta$ is a variable (and a central element), with a set of generators $\left\{x_{i j}, y_{i j}, z_{i} \mid\right.$ $1 \leq i \neq j \leq n\}$ subject to the following relations:

(1) $x_{i j}+x_{j i}=0, y_{i j}=y_{j i}$, for $i \neq j$,

(2) $x_{i j} x_{j k}=x_{i k} x_{i j}+x_{j k} x_{i k}+\beta x_{i k}$, for $1 \leq i<j<k \leq n$,

(3) $x_{i j} y_{j k}=y_{i k} x_{i j}+y_{j k} y_{i k}+\beta y_{i k}$, for $1 \leq i<j<k \leq n$,

(4) $x_{i k} y_{j k}=y_{j k} y_{i j}+y_{i j} x_{i k}+\beta y_{i j}$, for $1 \leq i<j<k \leq n$,

(5) $y_{i k} x_{j k}=x_{j k} y_{i j}+y_{i j} y_{i k}+\beta y_{i j}$, for $1 \leq i<j<k \leq n$, 
(6) $y_{i j} x_{i j}=z_{i} x_{i j}+y_{i j} z_{i}+\beta z_{i}$, for $i<j$,

(7) $x_{i j} z_{j}=y_{i j} x_{i j}+z_{j} y_{i j}+\beta y_{i j}$, for $i<j$.

Notice that when we set $\beta=0$ relations (2)-(5) of $\mathcal{S}\left(C_{n}\right)$ become relations (5)-(8) of $\mathcal{B}^{n c}\left(C_{n}\right)$, and if we combine relations (6) and (7) of $\mathcal{S}\left(C_{n}\right)$ we obtain relation (9) of $\mathcal{B}^{n c}\left(C_{n}\right)$. In some cases we will in fact simply work with the commutative counterpart of $\mathcal{B}^{n c}\left(C_{n}\right)$, namely $\mathcal{B}^{c}\left(C_{n}\right)$. Note that for $\beta=0$ the only difference between $\mathcal{B}^{c}\left(C_{n}\right)$ and $\mathcal{S}\left(C_{n}\right)$ is that relations (6) and (7) of $\mathcal{S}\left(C_{n}\right)$ are combined into a single relation $x_{i j} z_{j}=z_{i} x_{i j}+y_{i j} z_{i}+z_{j} y_{i j}$ in $\mathcal{B}^{c}\left(C_{n}\right)$.

We treat relations (2)-(7) of $\mathcal{S}\left(C_{n}\right)$ as reduction rules:

$$
\begin{gathered}
x_{i j} x_{j k} \rightarrow x_{i k} x_{i j}+x_{j k} x_{i k}+\beta x_{i k}, \\
x_{i j} y_{j k} \rightarrow y_{i k} x_{i j}+y_{j k} y_{i k}+\beta y_{i k}, \\
x_{i k} y_{j k} \rightarrow y_{j k} y_{i j}+y_{i j} x_{i k}+\beta y_{i j}, \\
y_{i k} x_{j k} \rightarrow x_{j k} y_{i j}+y_{i j} y_{i k}+\beta y_{i j}, \\
y_{i j} x_{i j} \rightarrow z_{i} x_{i j}+y_{i j} z_{i}+\beta z_{i}, \\
x_{i j} z_{j} \rightarrow y_{i j} x_{i j}+z_{j} y_{i j}+\beta y_{i j} .
\end{gathered}
$$

A reduced form of the monomial $m$ in variables $x_{i j}, y_{i j}, z_{k}, 1 \leq i<j \leq n, k \in$ $[n]$, in the algebra $\mathcal{S}\left(C_{n}\right)$ is a polynomial $P_{n}^{\mathcal{S}}$ obtained by successive applications of reductions (3)-(8) until no further reduction is possible, where we allow commuting any two variables. Requiring that $m$ is in variables $x_{i j}, y_{i j}, z_{k}, 1 \leq i<j \leq n, k \in$ $[n]$, is without loss of generality, since otherwise we can simply replace $x_{i j}$ with $-x_{j i}$ and $y_{i j}$ with $y_{j i}$. Note that the reduced forms are not necessarily unique. However we show in Section 7 that the number of monomials in a reduced form of a suitable monomial $m$ is independent of the order of the reductions performed.

\section{Commutative Reductions in terms of GraphS}

In this section we rephrase the reduction process described in Section 2 in terms of graphs. This view will be useful throughout the paper. We use the language of signed graphs. Signed graphs have appeared in the literature before, for example in Zaslavsky's and Reiner's work [Z1, Z2, R1, R2]. Their notation is not the same, and we use a notation closer to Reiner's. In particular, positive and negative edges in our notation mean something different than in Zaslavsky's language. We request the reader to read the definitions with full attention for this reason.

A signed graph $G$ on the vertex set $[n]$ is a multigraph with each edge labeled by + or - . All graphs in this paper are signed and in each of them the loops are labeled positive. We denote an edge with endpoints $i, j$ and sign $\epsilon \in\{+,-\}$ by $(i, j, \epsilon)$. Note that $(i, j, \epsilon)=(j, i, \epsilon)$. As a result, we drop the signs from the loops in the figures. A positive edge, that is, an edge labeled by + , is said to be positively incident, or, incident with a positive sign, to both of its endpoints. A negative edge is positively incident to its smaller vertex and negatively incident to its greater endpoint. We say that a graph is alternating if for any vertex $v \in V(G)$ the edges of $G$ incident to $v$ are incident to $v$ with the same sign. Note that this generalizes the type $A$ notion of alternating (unlabeled) graphs if we adopt the convention that each edge of an unlabeled graph is positively incident to its smaller vertex and negatively incident to its greater endpoint.

Think of a monomial $m \in \mathcal{S}\left(C_{n}\right)$ in variables $x_{i j}, y_{i j}, z_{k}, 1 \leq i<j \leq n, k \in[n]$, as a signed graph $G$ on the vertex set $[n]$ with a negative edge $(i, j,-)$ for each 
appearance of $x_{i j}$ in $m$ and with a positive edge $(i, j,+)$ for each appearance of $y_{i j}$ in $m$ and with a loop $(i, i,+)$ for each appearance of $z_{i}$ in $m$. Let $G^{\mathcal{S}}[m]$ denote this graph. It is straightforward to reformulate the reduction rules (3)-(8) in terms of reductions on graphs. If $m \in \mathcal{S}\left(C_{n}\right)$, then we replace each monomial $m$ in the reductions by corresponding graphs $G^{\mathcal{S}}[m]$.

Reduction rules for graphs:

Given a graph $G_{0}$ on the vertex set $[n]$ and $(i, j,-),(j, k,-) \in E\left(G_{0}\right)$ for some $i<j<k$, let $G_{1}, G_{2}, G_{3}$ be graphs on the vertex set $[n]$ with edge sets

$$
\begin{aligned}
& E\left(G_{1}\right)=E\left(G_{0}\right) \backslash\{(j, k,-)\} \cup\{(i, k,-)\}, \\
& E\left(G_{2}\right)=E\left(G_{0}\right) \backslash\{(i, j,-)\} \cup\{(i, k,-)\}, \\
& E\left(G_{3}\right)=E\left(G_{0}\right) \backslash\{(i, j,-)\} \backslash\{(j, k,-)\} \cup\{(i, k,-)\} .
\end{aligned}
$$

We say that $G_{0}$ reduces to $G_{1}, G_{2}, G_{3}$ under the reduction rule (9). See Figure 1 .

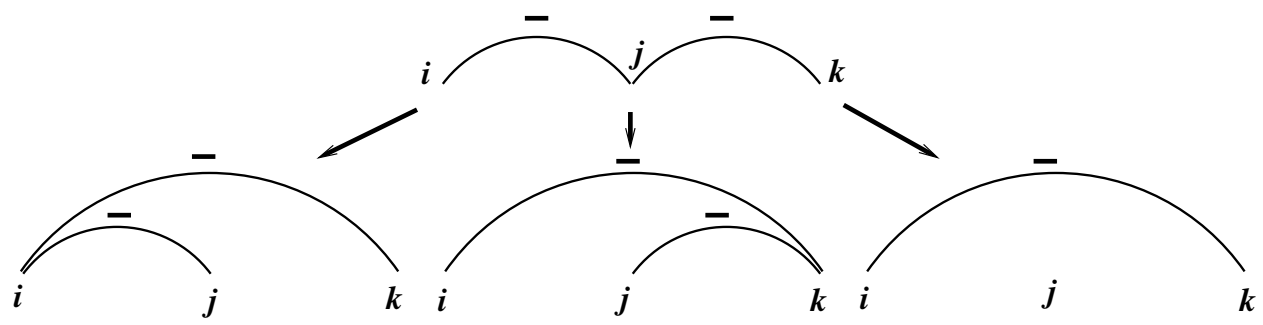

Figure 1. Reduction rule (9).

Given a graph $G_{0}$ on the vertex set $[n]$ and $(i, j,-),(j, k,+) \in E\left(G_{0}\right)$ for some $i<j<k$, let $G_{1}, G_{2}, G_{3}$ be graphs on the vertex set $[n]$ with edge sets

$$
\begin{aligned}
& E\left(G_{1}\right)=E\left(G_{0}\right) \backslash\{(j, k,+)\} \cup\{(i, k,+)\}, \\
& E\left(G_{2}\right)=E\left(G_{0}\right) \backslash\{(i, j,-)\} \cup\{(i, k,+)\}, \\
& E\left(G_{3}\right)=E\left(G_{0}\right) \backslash\{(i, j,-)\} \backslash\{(j, k,+)\} \cup\{(i, k,+)\} .
\end{aligned}
$$
2 .

We say that $G_{0}$ reduces to $G_{1}, G_{2}, G_{3}$ under the reduction rule (10). See Figure

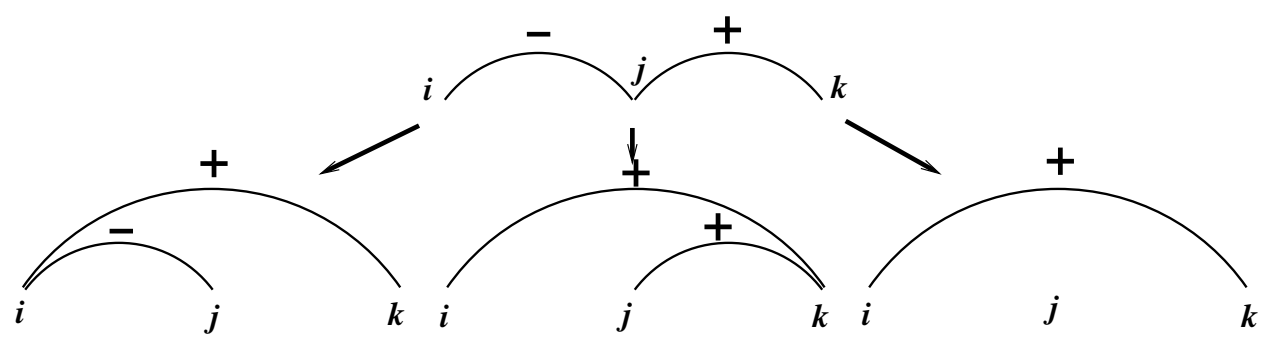

Figure 2. Reduction rule (10). 
Given a graph $G_{0}$ on the vertex set $[n]$ and $(i, k,-),(j, k,+) \in E\left(G_{0}\right)$ for some $i<j<k$, let $G_{1}, G_{2}, G_{3}$ be graphs on the vertex set [n] with edge sets

$$
\begin{aligned}
& E\left(G_{1}\right)=E\left(G_{0}\right) \backslash\{(j, k,+)\} \cup\{(i, j,+)\}, \\
& E\left(G_{2}\right)=E\left(G_{0}\right) \backslash\{(i, k,-)\} \cup\{(i, j,+)\}, \\
& E\left(G_{3}\right)=E\left(G_{0}\right) \backslash\{(i, k,-)\} \backslash\{(j, k,+)\} \cup\{(i, j,+)\} .
\end{aligned}
$$
3 .

We say that $G_{0}$ reduces to $G_{1}, G_{2}, G_{3}$ under the reduction rule (11). See Figure

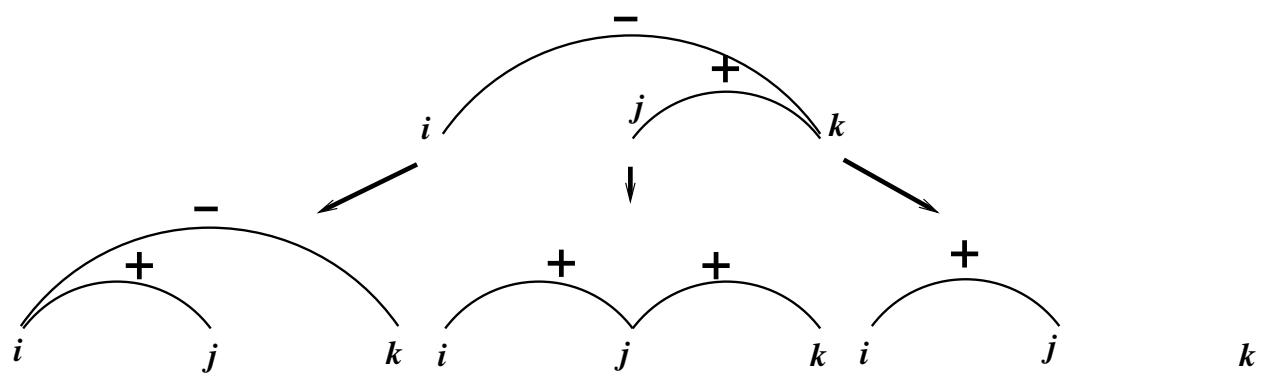

FiguRE 3. Reduction rule (11).

Given a graph $G_{0}$ on the vertex set $[n]$ and $(i, k,+),(j, k,-) \in E\left(G_{0}\right)$ for some $i<j<k$, let $G_{1}, G_{2}, G_{3}$ be graphs on the vertex set $[n]$ with edge sets

$$
\begin{aligned}
& E\left(G_{1}\right)=E\left(G_{0}\right) \backslash\{(j, k,-)\} \cup\{(i, j,+)\}, \\
& E\left(G_{2}\right)=E\left(G_{0}\right) \backslash\{(i, k,+)\} \cup\{(i, j,+)\}, \\
& E\left(G_{3}\right)=E\left(G_{0}\right) \backslash\{(i, k,+)\} \backslash\{(j, k,-)\} \cup\{(i, j,+)\} .
\end{aligned}
$$
4.

We say that $G_{0}$ reduces to $G_{1}, G_{2}, G_{3}$ under the reduction rule (12). See Figure

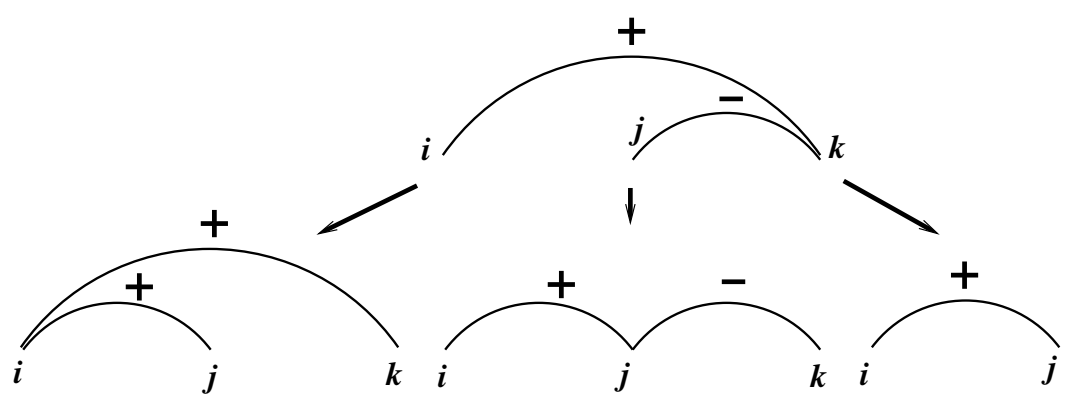

FiguRE 4. Reduction rule (12).

Given a graph $G_{0}$ on the vertex set $[n]$ and $(i, j,-),(i, j,+) \in E\left(G_{0}\right)$ for some $i<j$, let $G_{1}, G_{2}, G_{3}$ be graphs on the vertex set $[n]$ with edge sets

$$
\begin{aligned}
& E\left(G_{1}\right)=E\left(G_{0}\right) \backslash\{(i, j,+)\} \cup\{(i, i,+)\}, \\
& E\left(G_{2}\right)=E\left(G_{0}\right) \backslash\{(i, j,-)\} \cup\{(i, i,+)\}, \\
& E\left(G_{3}\right)=E\left(G_{0}\right) \backslash\{(i, j,+)\} \backslash\{(i, j,+)\} \cup\{(i, i,+)\} .
\end{aligned}
$$



5 .

We say that $G_{0}$ reduces to $G_{1}, G_{2}, G_{3}$ under the reduction rule (13). See Figure

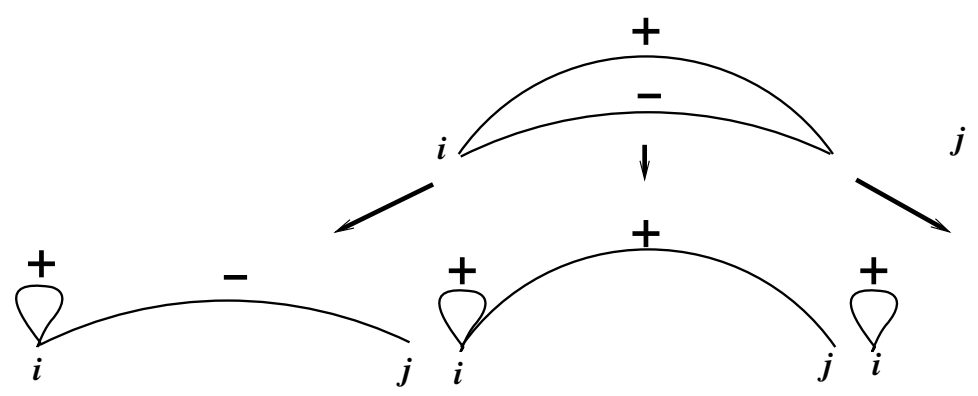

Figure 5. Reduction rule (13).

Given a graph $G_{0}$ on the vertex set $[n]$ and $(i, j,-),(j, j,+) \in E\left(G_{0}\right)$ for some $i<j$, let $G_{1}, G_{2}, G_{3}$ be graphs on the vertex set $[n+1]$ with edge sets

$$
\begin{aligned}
& E\left(G_{1}\right)=E\left(G_{0}\right) \backslash\{(j, j,+)\} \cup\{(i, j,+)\}, \\
& E\left(G_{2}\right)=E\left(G_{0}\right) \backslash\{(i, j,-)\} \cup\{(i, j,+)\}, \\
& E\left(G_{3}\right)=E\left(G_{0}\right) \backslash\{(j, j,+)\} \backslash\{(i, j,-)\} \cup\{(i, j,+)\} .
\end{aligned}
$$
6.

We say that $G_{0}$ reduces to $G_{1}, G_{2}, G_{3}$ under the reduction rule (14). See Figure

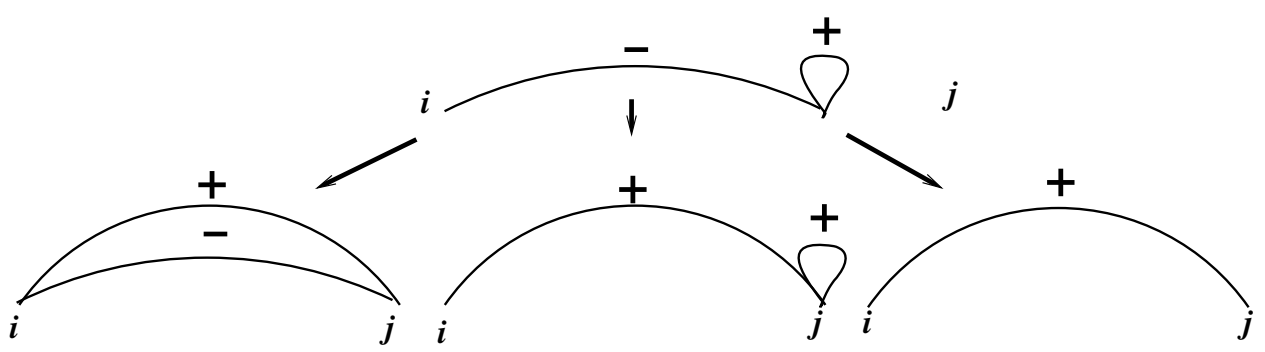

FiguRE 6. Reduction rule (14).

An $\mathcal{S}$-reduction tree $\mathcal{T}^{\mathcal{S}}$ for a monomial $m_{0}$, or equivalently, the graph $G^{\mathcal{S}}\left[m_{0}\right]$, is constructed as follows. The root of $\mathcal{T}^{\mathcal{S}}$ is labeled by $G^{\mathcal{S}}\left[m_{0}\right]$. A node $G^{\mathcal{S}}[m]$ in $\mathcal{T}^{\mathcal{S}}$ is a leaf if it is impossible to perform any of the above reductions on it. Otherwise a node $G^{\mathcal{S}}[m]$ in $\mathcal{T}^{\mathcal{S}}$ has three children, which depend on the choice of the edges of $G^{\mathcal{S}}[\mathrm{m}]$ on which we perform the reduction. E.g., if the reduction is performed on edges $(i, j,-),(j, k,-) \in E\left(G^{\mathcal{S}}[m]\right), i<j<k$, then the three children of the node $G_{0}=G^{\mathcal{S}}[\mathrm{m}]$ are labeled by the graphs $G_{1}, G_{2}, G_{3}$ as described by equation (91). For an example of an $\mathcal{S}$-reduction tree, see Figure 7 ,

Of course, given a graph we can also easily recover the corresponding monomial. Namely, given a graph $G$ on the vertex set $[n]$ we associate to it the monomial $m^{\mathcal{S}}[G]=m^{\mathcal{B}^{c}}[G]=\prod_{(i, j, \epsilon) \in E(G)} \mathrm{w}(i, j, \epsilon)$, where $\mathrm{w}(i, j,-)=x_{i j}$ for $i<j$, 


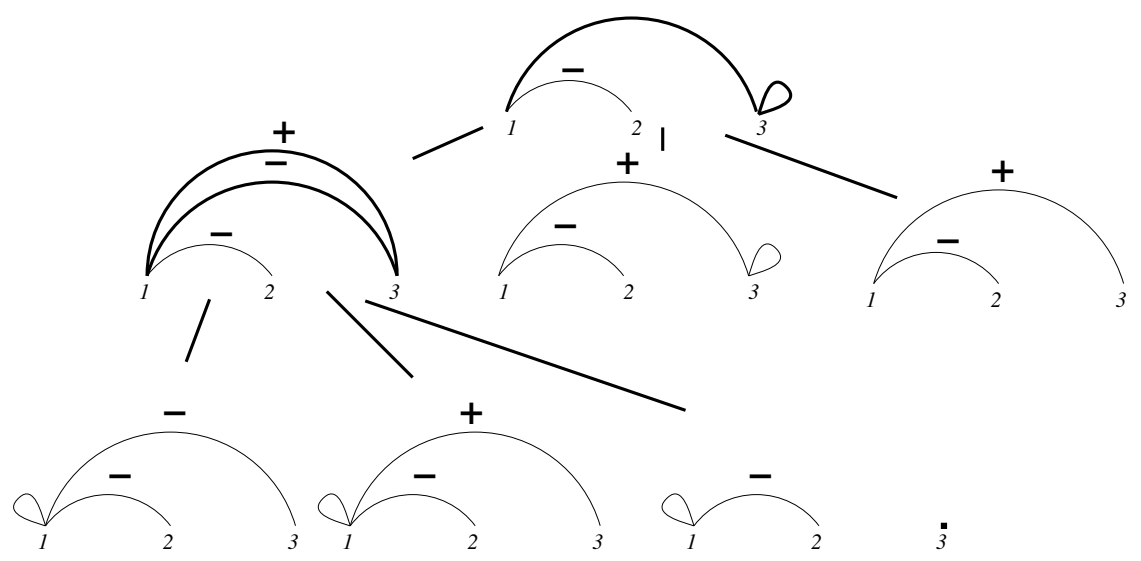

FiguRE 7. An $\mathcal{S}$-reduction tree with root corresponding to the monomial $x_{12} x_{13} z_{3}$. Summing the monomials corresponding to the graphs labeling the leaves of the reduction tree multiplied by suitable powers of $\beta$, we obtain a reduced form $P_{n}^{\mathcal{S}}$ of $x_{12} x_{13} z_{3}$, $P_{n}^{\mathcal{S}}=z_{1} x_{12} x_{13}+z_{1} x_{12} y_{13}+\beta z_{1} x_{12}+x_{12} y_{13} z_{3}+\beta x_{12} y_{13}$.

$\mathrm{w}(i, j,-)=x_{j i}$ for $i>j, \mathrm{w}(i, j,+)=y_{i j}$ and $\mathrm{w}(i, i,+)=z_{i}$. Summing the monomials corresponding to the graphs labeling the leaves of the reduction tree $\mathcal{T}^{\mathcal{S}}$ multiplied by suitable powers of $\beta$, we obtain a reduced form of $m_{0}$.

\section{Coned type $C$ Root polytopes}

Generalizing the terminology of [P Definition 12.1], a root polytope of type $C_{n}$ is the convex hull of the origin and some of the points $e_{i}-e_{j}, e_{i}+e_{j}$ and $2 e_{k}$ for $1 \leq i<j \leq n, k \in[n]$, where $e_{i}$ denotes the $i^{\text {th }}$ coordinate vector in $\mathbb{R}^{n}$. A very special root polytope is the full type $C_{n}$ root polytope

$$
\begin{aligned}
\mathcal{P}\left(C_{n}^{+}\right) & =\operatorname{ConvHull}\left(0, e_{i j}^{-}, e_{i j}^{+}, 2 e_{k} \mid 1 \leq i<j \leq n, k \in[n]\right) \\
& =\operatorname{ConvHull}\left(0, e_{i j}^{-}, 2 e_{k} \mid 1 \leq i<j \leq n, k \in[n]\right),
\end{aligned}
$$

where $e_{i j}^{-}=e_{i}-e_{j}$ and $e_{i j}^{+}=e_{i}+e_{j}$. We study a class of root polytopes including $\mathcal{P}\left(C_{n}^{+}\right)$, which we now discuss.

We use the notation $e_{i j}^{-}=e_{j i}^{-}=e_{i}-e_{j}$ for $i \leq j$ and $e_{i j}^{+}=e_{j i}^{+}=e_{i}+e_{j}$ for $i \leq j$. Furthermore, if $e=(i, j, \epsilon)$ is an edge of graph $G$, then $\mathrm{v}(e)=e_{i j}^{\epsilon}$. Define

$$
\begin{gathered}
\mathcal{V}_{G}=\left\{e_{i j}^{\epsilon} \mid(i, j, \epsilon) \in E(G)\right\}, \text { a set of vectors associated to } G ; \\
\mathcal{C}(G)=\left\langle\mathcal{V}_{G}\right\rangle:=\left\{\sum_{e_{i j}^{\epsilon} \in \mathcal{V}_{G}} c_{i j} e_{i j}^{\epsilon} \mid c_{i j} \geq 0\right\}, \text { the cone associated to } G ; \text { and } \\
\overline{\mathcal{V}}_{G}=\Phi^{+} \cap \mathcal{C}(G), \text { all the positive roots of type } C_{n} \text { contained in } \mathcal{C}(G),
\end{gathered}
$$

where $\Phi^{+}=\left\{e_{i j}^{-}, e_{i j}^{+}, 2 e_{k} \mid 1 \leq i<j \leq n, k \in[n]\right\}$ is the set of positive roots of type $C_{n}$. The idea to consider the positive roots of a root system inside a cone appeared earlier in Reiner's work [R1], [R2] on signed posets. Coned type $A_{n}$ root polytopes were studied in [M]. 
Define the transitive closure of a graph $G$ as

$$
\bar{G}=\left\{(i, j, \epsilon) \mid e_{i j}^{\epsilon} \in \overline{\mathcal{V}}_{G}\right\} .
$$

The transitive closure of a graph can be obtained by repeatedly adding edges as long as possible, with the following basic rules. If edges $(i, j,-)$ and $(j, k,-)$, $i<j<k$, are present, add edge $(i, k,-)$. If edges $(i, j,-)$ and $(j, k,+), i<j<k$, are present, add edge $(i, k,+)$. If edges $(i, j,-)$ and $(k, j,+), k<i<j$ or $i<k<j$, are present, add edge $(k, j,+)$. If edges $(i, j,-)$ and $(i, j,+), i<j$, are present, add edge $(i, i,+)$. If edges $(i, j,-)$ and $(j, j,+), i<j$, are present, add edge $(i, j,+)$.

The root polytope $\mathcal{P}(G)$ associated to the graph $G$ is

$$
\mathcal{P}(G)=\operatorname{ConvHull}\left(0, e_{i j}^{\epsilon} \mid(i, j, \epsilon) \in \bar{G}\right) .
$$

The root polytope $\mathcal{P}(G)$ associated to the graph $G$ can also be defined as

$$
\mathcal{P}(G)=\mathcal{P}\left(C_{n}^{+}\right) \cap \mathcal{C}(G) .
$$

The equivalence of these two definitions is proved in Lemma 8 in Section 6 .

Note that $\mathcal{P}\left(C_{n}^{+}\right)=\mathcal{P}\left(P^{l}\right)$ for the graph $P^{l}=([n],\{(n, n,+),(i, i+1,-) \mid i \in$ $[n-1]\})$. While the choice of $G$ such that $\mathcal{P}\left(C_{n}^{+}\right)=\mathcal{P}(G)$ is not unique, it becomes unique if we require that $G$ is minimal, that is, for no edge $(i, j, \epsilon) \in E(G)$ can the corresponding vector $e_{i j}^{\epsilon}$ be written as a nonnegative linear combination of the vectors corresponding to the edges $E(G) \backslash\{(i, j, \epsilon)\}$. Graph $P^{l}$ is minimal.

We can describe the vertices in $\overline{\mathcal{V}}_{G}$ in terms of paths in $G$. A playable route $P$ of a graph $G$ is a path (with a sign compatibility at each vertex) $\left(i_{1}, j_{1}, \epsilon_{1}\right), \ldots$, $\left(i_{l}, j_{l}, \epsilon_{l}\right) \in E(G), j_{k}=i_{k+1}$ for $k \in[l-1]$, such that $\left(i_{k}, j_{k}, \epsilon_{k}\right)$ and $\left(i_{k+1}, j_{k+1}\right.$, $\left.\epsilon_{k+1}\right), k \in[l-1]$, are incident to $j_{k}=i_{k+1}$ with opposite signs. For a playable route of $G, e_{i_{1}, j_{1}}^{\epsilon_{1}}+\cdots+e_{i_{l}, j_{l}}^{\epsilon_{l}} \in \Phi^{+}$.

A playable pair $\left(P_{1}, P_{2}\right)$ in a graph $G$ is a pair of playable routes $\left(i_{1}, j_{1}, \epsilon_{1}\right), \ldots$, $\left(i_{l}, j_{l}, \epsilon_{l}\right)$ and $\left(i_{1}^{\prime}, j_{1}^{\prime}, \epsilon_{1}^{\prime}\right), \ldots,\left(i_{l^{\prime}}^{\prime}, j_{l^{\prime}}^{\prime}, \epsilon_{l^{\prime}}^{\prime}\right)$ such that $i_{1}=j_{l}$ and $i_{1}^{\prime}=j_{l^{\prime}}^{\prime}$. It follows that $\frac{1}{2}\left(e_{i_{1}, j_{1}}^{\epsilon_{1}}+\cdots+e_{i_{l}, j_{l}}^{\epsilon_{l}}\right)+\frac{1}{2}\left(e_{i_{1}^{\prime}, j_{1}^{\prime}}^{\epsilon_{1}^{\prime}}+\cdots+e_{i_{l^{\prime}}, j_{l^{\prime}}^{\prime}}^{\epsilon_{l^{\prime}}}\right) \in \Phi^{+}$.

Define a map $\phi$ from the playable routes and playable pairs to $\Phi^{+}$as follows:

$$
\begin{aligned}
\phi(P) & =e_{i_{1}, j_{1}}^{\epsilon_{1}}+\cdots+e_{i_{l}, j_{l}}^{\epsilon_{l}}, \text { where } P \text { is the playable route above, } \\
\phi\left(P_{1}, P_{2}\right) & =\frac{1}{2}\left(e_{i_{1}, j_{1}}^{\epsilon_{1}}+\cdots+e_{i_{l}, j_{l}}^{\epsilon_{l}}\right)+\frac{1}{2}\left(e_{i_{1}^{\prime}, j_{1}^{\prime}}^{\epsilon_{1}^{\prime}}+\cdots+e_{i_{l^{\prime}}, j_{l^{\prime}}^{\prime}}^{\epsilon_{\prime^{\prime}}}\right), \\
& \text { where }\left(P_{1}, P_{2}\right) \text { is the playable pair above. }
\end{aligned}
$$

Proposition 1. Let $G$ be a graph on the vertex set $[n]$. Any $v \in \overline{\mathcal{V}}_{G}$ is $v=\phi(P)$ or $v=\phi\left(P_{1}, P_{2}\right)$ for some playable route $P$ or playable pair $\left(P_{1}, P_{2}\right)$ of $G$. If the set of vectors $\mathcal{V}_{G}$ is linearly independent, then the correspondence between playable routes and pairs of $G$ and vectors in $\overline{\mathcal{V}}_{G}$ is a bijection.

The proof of Proposition 1 appears in Section 5 .

Define

$$
\mathcal{L}_{n}=\left\{G=([n], E(G)) \mid \mathcal{V}_{G} \text { is a linearly independent set }\right\}
$$

and

$$
\mathcal{L}\left(C_{n}^{+}\right)=\left\{\mathcal{P}(G) \mid G \in \mathcal{L}_{n}\right\} \text {, the set of type } C_{n} \text { coned root polytopes }
$$

with linearly independent generators. Since all polytopes in this paper are coned root polytopes with linearly independent generators, we simply refer to them as coned root polytopes. 
The next lemma characterizes graphs $G$ which belong to $\mathcal{L}_{n}$; a version of it appears in [F, p. 42].

Lemma $2([\mathrm{~F}$, p. 42$])$. A graph $G$ on the vertex set $[n]$ belongs to $\mathcal{L}_{n}$ if and only if each connected component of $G$ is a tree or a graph whose unique simple cycle has an odd number of positively labeled edges.

The full root polytope $\mathcal{P}\left(C_{n}^{+}\right) \in \mathcal{L}\left(C_{n}^{+}\right)$, since the graph $P^{l} \in \mathcal{L}_{n}$ by Lemma 2 , We show below how to obtain central triangulations for all polytopes $\mathcal{P} \in \mathcal{L}\left(C_{n}^{+}\right)$. A central triangulation of a $d$-dimensional root polytope $\mathcal{P}$ is a collection of $d$-dimensional simplices with disjoint interiors whose union is $\mathcal{P}$, the vertices of which are vertices of $\mathcal{P}$ and the origin is a vertex of all of them. Depending on the context we at times take the intersections of these maximal simplices to be part of the triangulation.

We now state the crucial lemma which relates root polytopes and the algebras $\mathcal{B}^{n c}\left(C_{n}\right), \mathcal{B}^{c}\left(C_{n}\right)$ and $\mathcal{S}\left(C_{n}\right)$ defined in Section 2 ,

Lemma 3 (Reduction Lemma). Given a graph $G_{0} \in \mathcal{L}_{n}$ with d edges, let $G_{1}, G_{2}, G_{3}$ be as described by any one of the equations (9) -(14). Then $G_{1}, G_{2}, G_{3} \in \mathcal{L}_{n}$,

$$
\mathcal{P}\left(G_{0}\right)=\mathcal{P}\left(G_{1}\right) \cup \mathcal{P}\left(G_{2}\right),
$$

where all polytopes $\mathcal{P}\left(G_{0}\right), \mathcal{P}\left(G_{1}\right), \mathcal{P}\left(G_{2}\right)$ are d-dimensional, and

$$
\mathcal{P}\left(G_{3}\right)=\mathcal{P}\left(G_{1}\right) \cap \mathcal{P}\left(G_{2}\right) \text { is }(d-1) \text {-dimensional. }
$$

What the Reduction Lemma really says is that performing a reduction on the graph $G_{0} \in \mathcal{L}_{n}$ is the same as "cutting" the $d$-dimensional polytope $\mathcal{P}\left(G_{0}\right)$ into two $d$-dimensional polytopes $\mathcal{P}\left(G_{1}\right)$ and $\mathcal{P}\left(G_{2}\right)$, whose vertex sets are subsets of the vertex set of $\mathcal{P}\left(G_{0}\right)$, whose interiors are disjoint, whose union is $\mathcal{P}\left(G_{0}\right)$, and whose intersection is a facet of both. We prove the Reduction Lemma in Section 6 .

\section{Characterizing the Vertices of CONED RoOT POlytopes}

In this section we prove Proposition 1, which characterizes the vertices of any root polytope $\mathcal{P}(G)$. We start by proving the statement for connected $G \in \mathcal{L}_{n}$.

Proposition 4. Let $G \in \mathcal{L}_{n}$ be a connected graph. The correspondence between playable routes of $G$ and vectors in $\overline{\mathcal{V}}_{G}$, given by

$$
\phi: P=\left\{\left(i_{1}, j_{1}, \epsilon_{1}\right),\left(i_{2}, j_{2}, \epsilon_{2}\right), \ldots,\left(i_{l}, j_{l}, \epsilon_{l}\right)\right\} \mapsto e_{i_{1}, j_{1}}^{\epsilon_{1}}+\cdots+e_{i_{l}, j_{l}}^{\epsilon_{l}},
$$

is a bijection.

Denote by $\left[e_{i}\right] w$ the coefficient of $e_{i}$ when $w \in \mathbb{R}^{n}$ is expressed in terms of the standard basis $e_{1}, \ldots, e_{n}$ of $\mathbb{R}^{n}$.

Proof of Proposition 4. Given a playable route $P$ of $G, \phi(P) \in \overline{\mathcal{V}}_{G}$ by definition. It remains to show that for each vertex $v \in \overline{\mathcal{V}}_{G}$ there exists a playable route $P$ in $G$ such that $v=\phi(P)$. The uniqueness of such a route follows from the linear independence of the set of vectors $\mathcal{V}_{G}$ for $G \in \mathcal{L}_{n}$.

Consider $v \in \overline{\mathcal{V}}_{G}$. Then $v=e_{i} \pm e_{j}$, for some $1 \leq i<j \leq n$, or $v=2 e_{k}=e_{k}+e_{k}$, for $k \in[n]$, and

$$
v=\sum_{e \in E(G)} c_{e} \mathrm{v}(e), \text { for some real } c_{e} \geq 0
$$


Let $H=\left([n],\left\{e \in E(G) \mid c_{e} \neq 0\right\}\right)$. Observe that $H$ has at most one connected component containing edges. This follows since a connected $G \in \mathcal{L}_{n}$ contains at most one simple cycle, and if there were two connected components of $H$, one would be a tree contributing at least two nonzero coordinates to the right hand side of (18) and each connected component containing edges contributes at least one nonzero coordinate to the right hand side of (18). But, the left hand side of (18) has one or two nonzero coordinates.

If $k$ is a leaf of $H$, then $\left[e_{k}\right] v \neq 0$. Therefore, $H$ can have at most two leaves. We consider three cases depending on the number of leaves $H$ has: $0,1,2$. In all cases we show that there exists a playable route $P$ of $G$ with all its edges among the edges of $H$, such that $\phi(P)=v$, yielding the desired conclusion.

Case 1. $H$ has 0 leaves. Since $H \subset G \in \mathcal{L}_{n}$, it follows that $H$ is a simple cycle. Relabel the vertices of the cycle so that $H$ is now a graph on $[m]$. Then $i=1$ since 1 only has edges positively incident to it. Regardless of which vertex of $H$ is $j>1$, there is a playable route $P$ starting at vertex $i$ and ending at $j$ such that $\phi(P)=v$.

Case 2. $H$ has 1 leaf. Then $H$ is a union of a simple cycle $C$ and a simple path $Q$. Relabel the vertices of $H$ so that it is a graph on the vertex set $[m]$. Let $l$ be the leftmost vertex of the cycle $C$ of $H$ and let $p$ be the vertex in common with $C$ and $Q$. Let $k$ be the unique leaf.

If $l \neq p$, then $\{i, j\}=\{l, k\}$. Thus, at least one of the edges of $C$ incident to $p$ is incident with an opposite sign to $p$ than the edge of $Q$ incident to $p$. Therefore, the edges on the path from $l$ to $p$ through the edge that is incident to $p$ in $C$ with the opposite sign to that of the edge of $Q$, and then the edges of path $Q$ form a playable route $P$ such that $\phi(P)=v$.

If $l=p$, then we consider two possibilities, depending on whether $l \notin\{i, j\}$ or $l \in\{i, j\}$. If $l \notin\{i, j\}$, then $i=k=1$ and $l \neq j$. If $j \in C$, then the edges of $Q$ (from 1 to $l$ ) and the edges on the path from $l$ to $j$ through the edge that is incident to $j$ in $C$ with the sign of $e_{j}$ in $v$ make up a playable route $P$ with $\phi(P)=v$. If $j \in Q$, however, then either the edges on the path from $i$ to $j$ along $Q$ make up a playable route $P$ with $\phi(P)=v$, or the edges of $Q$ (from 1 to $l$ ) and the edges of $C$ and then the edges on the path from $l$ to $j$ make up a playable route $P$ with $\phi(P)=v$.

If $l=p$ and $l \in\{i, j\}$, then either $i=l$ or $j=l$. If $i=l$, then the edges on the path $Q$ from $l=1$ to $j=k$ make up a playable route $P$ with $\phi(P)=v$. On the other hand, if $j=l$, then $i=1$ and if the edge of $Q$ is incident to $l$ with the same sign as that of the sign of $e_{j}$ in $v$, then the edges of $Q$ make up a playable route $P$ with $\phi(P)=v$. If, however, that sign is different, then it must be that $\left[e_{j}\right] v=1$, in which case all edges of $H$ (suitably ordered) make up a playable route $P$ with $\phi(P)=v$.

Case 3. $H$ has 2 leaves. Then $H$ could be a path, or a union of a simple cycle $C$ and two disjoint paths $Q_{1}, Q_{2}$ attached to $C$ at vertices $p_{1} \neq p_{2}$, or a union of a cycle $C$ and a tree $T$ with two leaves attached to $C$ at $t$. As in cases 1 and 2, in each case we can identify a playable route by inspection. We omit the details here.

Proposition 4 yields a characterization of the vertices of $\mathcal{P}(G)$ for a connected $G \in \mathcal{L}_{n}$.

Proposition 5. Let $G \in \mathcal{L}_{n}$. The map $\phi$ defined by (17) is a one-to-one correspondence between playable routes and playable pairs of $G$ and the vectors in $\overline{\mathcal{V}}_{G}$. 
Proof. The proof is almost identical to that of Proposition 4. The only difference is that the graph $H$ defined in the proof of Proposition 4 could have two connected components containing edges. The case of $H$ with one connected component containing edges is the same as in the proof of Proposition 4.

Let the two connected components of $H$ containing edges be $H_{1}$ and $H_{2}$. Then, $H_{1}$ and $H_{2}$ each contribute exactly one coordinate with a nonzero coefficient, and thus each of them is a union of a simple cycle (since $G \in \mathcal{L}_{n}$ ) and a possibly empty simple path. The edges of $H_{1}$ and $H_{2}$, in a suitable order, constitute playable pairs.

Proposition 6. For any graph $G$ the set of vectors $\overline{\mathcal{V}}_{G}$ is the image of playable routes and pairs of $G$ under the map $\phi$ defined by (17).

Proof. Let $P(G)=\operatorname{ConvHull}\left(0, e_{i j}^{\epsilon} \mid e_{i j}^{\epsilon} \in \mathcal{V}_{G}\right)$, and let $\Delta$ be a central triangulation of $P(G)$. For each $\sigma \in \Delta$ we define $\mathcal{C}(\sigma)=\mathcal{C}\left(G^{\prime}\right)$, where the vertex set of $\sigma$ is $\left\{0, e_{i j}^{\epsilon} \mid(i, j, \epsilon) \in G^{\prime}\right\}, G^{\prime} \subset G$ and $G^{\prime} \in \mathcal{L}_{n}$. Then,

$$
\overline{\mathcal{V}}_{G} \subset \mathcal{C}(G)=\bigcup_{\sigma \in \Delta} \mathcal{C}(\sigma) .
$$

Thus, any $v \in \overline{\mathcal{V}}_{G}$ belongs to some $\mathcal{C}\left(G^{\prime}\right)$. Therefore, $v \in \overline{\mathcal{V}}_{G^{\prime}}$, for $G^{\prime} \in \mathcal{L}_{n}$, $G^{\prime} \subset G$. By Proposition 5 there is a playable route $P$ or pair $\left(P_{1}, P_{2}\right)$ in $G^{\prime}$, such that $v=\phi(P)$ or $v=\phi\left(P_{1}, P_{2}\right)$. But all playable routes and pairs of $G^{\prime}$ are also playable routes and pairs of $G$.

Propositions 4 5 and 6 imply Proposition 1.

\section{The proof of the Reduction Lemma}

This section is devoted to proving the Reduction Lemma (Lemma 3). As we shall see in Section 7 the Reduction Lemma is the "secret force" that makes everything fall into its place for coned root polytopes. We start by characterizing the root polytopes which are simplices, then in Lemma 8 we prove that equations (15) and (16) are equivalent definitions for the root polytope $\mathcal{P}(G)$, and finally we prove the Cone Reduction Lemma (Lemma 9), which, together with Lemma 8 implies the Reduction Lemma.

Lemma 7. For a graph $G$ on the vertex set $[n]$ with $d$ edges, the polytope $\mathcal{P}(G)$ as defined by (15) is a simplex if and only if $G$ is alternating and $G \in \mathcal{L}_{n}$.

Proof. It follows from equation (15) that for a minimal graph $G$ the polytope $\mathcal{P}(G)$ is a simplex if and only if the vectors corresponding to the edges of $G$ are linearly independent and $\mathcal{C}(G) \cap \Phi^{+}=\mathcal{V}_{G}$.

The vectors corresponding to the edges of $G$ are linearly independent if and only if $G \in \mathcal{L}_{n}$. By Proposition $1 \mathcal{C}(G) \cap \Phi^{+}=\mathcal{V}_{G}$ if and only if $G$ contains no edges incident to a vertex $v \in V(G)$ with opposite signs, i.e., $G$ is alternating.

Lemma 8. For any graph $G$ on the vertex set $[n]$,

$$
\operatorname{ConvHull}\left(0, e_{i j}^{\epsilon} \mid(i, j, \epsilon) \in \bar{G}\right)=\mathcal{P}\left(C_{n}^{+}\right) \cap \mathcal{C}(G) .
$$

Proof. For a graph $H$ on the vertex set $[n]$, let $\sigma(H)=\operatorname{ConvHull}\left(0, e_{i j}^{\epsilon} \mid(i, j, \epsilon) \epsilon\right.$ $H)$. Then, $\sigma(\bar{G})=\operatorname{ConvHull}\left(0, e_{i j}^{\epsilon} \mid(i, j, \epsilon) \in \bar{G}\right)$. Let $\sigma(\bar{G})$ be a $d$-dimensional polytope for some $d \leq n$ and consider any central triangulation of it: $\sigma(\bar{G})=$ 
$\bigcup_{F \in \mathcal{F}} \sigma(F)$, where $\{\sigma(F)\}_{F \in \mathcal{F}}$ is a set of $d$-dimensional simplices with disjoint interiors, $E(F) \subset E(\bar{G}), F \in \mathcal{F}$. Since $\sigma(\bar{G})=\bigcup_{F \in \mathcal{F}} \sigma(F)$ is a central triangulation, it follows that $\sigma(F)=\sigma(\bar{G}) \cap \mathcal{C}(F)$, for $F \in \mathcal{F}$, and $\mathcal{C}(G)=\bigcup_{F \in \mathcal{F}} \mathcal{C}(F)$.

Since $\sigma(F), F \in \mathcal{F}$, is a $d$-dimensional simplex, it follows that $F \in \mathcal{L}_{n}$ and has $d$ edges. Furthermore, $F \in \mathcal{F}$ is alternating, as otherwise there are edges $\left(i, j, \epsilon_{1}\right),\left(j, k, \epsilon_{2}\right) \in E(F) \subset E(\bar{G})$ incident to $j$ with opposite signs, and while $e_{i j}^{\epsilon_{1}}+e_{j k}^{\epsilon_{2}} \in \sigma(\bar{G}) \cap \mathcal{C}(F), e_{i j}^{\epsilon_{1}}+e_{j k}^{\epsilon_{2}} \notin \sigma(F)$, contradicting that $\bigcup_{F \in \mathcal{F}} \sigma(F)$ is a central triangulation of $\sigma(\bar{G})$. Thus, $\bar{F}=F$, and $\sigma(F)=\sigma(\bar{F})$. It is clear that $\sigma(\bar{F})=$ $\operatorname{ConvHull}\left(0, e_{i j}^{\epsilon} \mid(i, j, \epsilon) \in \bar{F}\right) \subset \mathcal{P}\left(C_{n}^{+}\right) \cap \mathcal{C}(F), F \in \mathcal{F}$. Since if $x=\left(x_{1}, \ldots, x_{n+1}\right)$ is in the facet of $\sigma(\bar{F})$ opposite the origin, then $\left|x_{1}\right|+\cdots+\left|x_{n+1}\right|=2$ and for any point $x=\left(x_{1}, \ldots, x_{n+1}\right) \in \mathcal{P}\left(C_{n}^{+}\right),\left|x_{1}\right|+\cdots+\left|x_{n+1}\right| \leq 2$ it follows that $\mathcal{P}\left(C_{n}^{+}\right) \cap \mathcal{C}(F) \subset \sigma(\bar{F})$. Thus, $\sigma(\bar{F})=\mathcal{P}\left(C_{n}^{+}\right) \cap \mathcal{C}(F)$. Finally, ConvHull $\left(0, e_{i j}^{\epsilon} \mid\right.$ $(i, j, \epsilon) \in \bar{G})=\sigma(\bar{G})=\bigcup_{F \in \mathcal{F}} \sigma(F)=\bigcup_{F \in \mathcal{F}} \sigma(\bar{F})=\bigcup_{F \in \mathcal{F}}\left(\mathcal{P}\left(C_{n}^{+}\right) \cap \mathcal{C}(F)\right)=$ $\mathcal{P}\left(C_{n}^{+}\right) \cap\left(\bigcup_{F \in \mathcal{F}} \mathcal{C}(F)\right)=\mathcal{P}\left(C_{n}^{+}\right) \cap \mathcal{C}(G)$ as desired.

Lemma 9 (Cone Reduction Lemma). Given a graph $G_{0} \in \mathcal{L}_{n}$ with $d$ edges, let $G_{1}, G_{2}, G_{3}$ be the graphs described by any one of the equations (9)-(14). Then $G_{1}, G_{2}, G_{3} \in \mathcal{L}_{n}$,

$$
\mathcal{C}\left(G_{0}\right)=\mathcal{C}\left(G_{1}\right) \cup \mathcal{C}\left(G_{2}\right)
$$

where all cones $\mathcal{C}\left(G_{0}\right), \mathcal{C}\left(G_{1}\right), \mathcal{C}\left(G_{2}\right)$ are d-dimensional, and

$$
\mathcal{C}\left(G_{3}\right)=\mathcal{C}\left(G_{1}\right) \cap \mathcal{C}\left(G_{2}\right) \text { is }(d-1) \text {-dimensional. }
$$

The proof of Lemma 9 is the same as that of the Cone Reduction Lemma in the type $A_{n}$ case; see [M, Lemma 7].

Proof of the Reduction Lemma (Lemma 3). This is a straightforward corollary of Lemmas 8 and 9

\section{Volumes of RoOT POLYTOPES AND The Number of MONOMials IN REDUCED FORMS}

In this section we use the Reduction Lemma to establish the link between the volumes of root polytopes and the number of monomials in reduced forms. In fact we shall see that if we know either of these quantities, we also know the other.

Proposition 10. Let $G_{0} \in \mathcal{L}_{n}$ be a connected graph on the vertex set $[n]$ with $n$ edges, and let $\mathcal{T}^{\mathcal{S}}$ be an $\mathcal{S}$-reduction tree with root labeled $G_{0}$. Then,

$$
\operatorname{vol}_{n}\left(\mathcal{P}\left(G_{0}\right)\right)=\frac{2 f\left(G_{0}\right)}{n !},
$$

where $f\left(G_{0}\right)$ denotes the number of leaves of $\mathcal{T}^{\mathcal{S}}$ labeled by graphs with $n$ edges.

Proof. By the Reduction Lemma (Lemma 3), $\operatorname{vol}_{n}\left(\mathcal{P}\left(G_{0}\right)\right)=\sum_{G} \operatorname{vol}_{n}(\mathcal{P}(G))$, where $G$ runs over the leaves of $\mathcal{T}^{\mathcal{S}}$ labeled by graphs with $n$ edges. We now prove that for each $G$ with $n$ edges labeling a leaf of $\mathcal{T}^{\mathcal{S}}$ with root labeled $G_{0}$, $\operatorname{vol}_{n}(\mathcal{P}(G))=\frac{2}{n !}$. Since $G_{0} \in \mathcal{L}_{n}$ is a connected graph on the vertex set $[n]$ with $n$ edges, so are all its successors with $n$ edges. If $G$ labels a leaf of $\mathcal{T}^{\mathcal{S}}$, then $G$ satisfies the conditions of Lemma 7 Thus, $\mathcal{P}(G)$ is a simplex.

The volume of $\mathcal{P}(G)$ can be calculated by calculating the determinant $\operatorname{det}(M)$ of the matrix $M$ whose rows are the vectors $\mathrm{v}(e), e \in E(G)$, written in the standard basis. If $v \in[n]$ is a vertex of degree 1 in $G$, then the $v^{\text {th }}$ column contains a single 1 
or -1 in the row corresponding to the edge incident to $v$. Let this row be the $v_{r}{ }^{\text {th }}$. Delete the $v^{\text {th }}$ column and $v_{r}^{\text {th }}$ row from $M$ and delete the edge incident to $v$ in $G$ obtaining a new graph. Successively identify the leaves in the new graphs and delete the corresponding columns and rows from their matrices until we obtain a graph $C$ that is a simple cycle and the corresponding matrix $M^{\prime}$. The rows of $M^{\prime}$ are the vectors $\mathrm{v}(e), e \in E(C)$. By Laplace expansion, $|\operatorname{det}(M)|=\left|\operatorname{det}\left(M^{\prime}\right)\right|$. Since $G \in \mathcal{L}_{n}$, so is $C \in \mathcal{L}_{n}$. Thus, $\operatorname{det}\left(M^{\prime}\right) \neq 0$. Expand $M^{\prime}$ by any of its rows obtaining matrices $M_{1}$ and $M_{2}$. Then we get $\left|\operatorname{det}\left(M^{\prime}\right)\right|=\left|\operatorname{det}\left(M_{1}\right)\right|+\left|\operatorname{det}\left(M_{2}\right)\right|=2$, since both $M_{1}$ and $M_{2}$ are such that their entries are all 0,1 or -1 , each row (column) except one has exactly two nonzero entries, and the remaining one exactly one nonzero entry. Thus, $\operatorname{vol}_{n}(\mathcal{P}(G))=\operatorname{det}(M) / n !=2 / n$ !

A general version of Proposition 10 can be proved for any connected $G_{0} \in \mathcal{L}_{n}$ using the following lemma.

Lemma 11. Let $G \in \mathcal{L}_{n}$ be an alternating graph on the vertex set $[n]$ with $d$ edges, with $c$ connected components of which $k \leq c$ contain simple cycles. Then,

$$
\operatorname{vol}_{d}(\mathcal{P}(G))=\frac{2^{k}}{d !}
$$

Proof. Let $M^{a}$ be the matrix whose rows are the vectors $e_{i j}^{\epsilon},(i, j, \epsilon) \in E(G)$, written in the standard basis. Matrix $M^{a}$ is a $d \times n$ matrix. The rows and columns of $M^{a}$ can be rearranged so that it has a block form in which the blocks $B_{1}, \ldots, B_{c}$ on the diagonal correspond to the connected components of $G$, while all other blocks are 0 . Since $G \in \mathcal{L}_{n}$ satisfies the conditions of Lemma $7, \mathcal{P}(G)$ is a $\operatorname{simplex,} \operatorname{vol}_{d}(\mathcal{P}(G)) \neq$ 0 and $\operatorname{vol}_{d}(\mathcal{P}(G))$ can be calculated by dropping some $n-d$ columns of $M^{a}$ such that the resulting matrix $M$ has nonzero determinant. Then, $\operatorname{vol}_{d}(\mathcal{P}(G))=|\operatorname{det}(M)| / d$ ! . Drop a column $b_{i}$ from the block matrix $B_{i}$ if the block $B_{i}$ corresponds to a tree on $m$ vertices, obtaining matrix $B_{i}^{\prime}$ with nonzero determinant. Then, $\left|\operatorname{det}\left(B_{i}^{\prime}\right)\right|=1$. If $B_{i}$ corresponds to a connected component of $G_{0}$ with $m$ vertices and $m$ edges, then $B_{i}^{\prime}=B_{i}$ and $\left|\operatorname{det}\left(B_{i}\right)\right|=2$. Since there are $n-d$ connected components which are trees, if we drop the columns $b_{i}$ from $M^{a}$ for all blocks $B_{i}$ corresponding to a tree obtaining a matrix $M$, then $\operatorname{vol}_{d}(\mathcal{P}(G))=\frac{|\operatorname{det}(M)|}{d !}$. Since $M$ has a special block form with blocks $B_{i}^{\prime}$ along the diagonal and zeros otherwise, we have that $|\operatorname{det}(M)|=\left|\prod_{i=1}^{c} \operatorname{det}\left(B_{i}^{\prime}\right)\right|=2^{k}$.

Proposition 12. Let $G_{0} \in \mathcal{L}_{n}$ be a graph on the vertex set $[n]$ with $d$ edges, with c connected components of which $k \leq$ contain cycles. Let $\mathcal{T}^{\mathcal{S}}$ be an $\mathcal{S}$-reduction tree with root labeled $G_{0}$. Then,

$$
\operatorname{vol}_{d}\left(\mathcal{P}\left(G_{0}\right)\right)=\frac{2^{k} f\left(G_{0}\right)}{d !}
$$

where $f\left(G_{0}\right)$ denotes the number of leaves of $\mathcal{T}^{\mathcal{S}}$ labeled by graphs with $d$ edges.

The proof of Proposition 12 proceeds analogously to Proposition 10, in view of Lemma 11.

Corollary 13. Let $G_{0} \in \mathcal{L}_{n}$ and let $m^{\mathcal{S}}\left[G_{0}\right]$ be the monomial corresponding to it. Then for any reduced form $P_{n}^{\mathcal{S}}$ of $m^{\mathcal{S}}\left[G_{0}\right]$, the value of $P_{n}^{\mathcal{S}}\left(x_{i j}=y_{i j}=z_{i}=1, \beta=\right.$ 0 ) is independent of the order of reductions performed. 
Proof. Note that $P_{n}^{\mathcal{S}}\left(x_{i j}=y_{i j}=1, \beta=0\right)=f\left(G_{0}\right)$, as defined in Proposition 12 , Since $\operatorname{vol}_{d}\left(\mathcal{P}\left(G_{0}\right)\right)$ is only dependent on $G_{0}$, the value of $P_{n}^{\mathcal{S}}\left(x_{i j}=y_{i j}=z_{i}=1, \beta=\right.$ $0)$ is independent of the particular reductions performed.

With analogous methods, the following proposition about reduced forms in $\mathcal{B}^{c}\left(C_{n}\right)$ can also be proved.

Proposition 14. Let $G_{0} \in \mathcal{L}_{n}$ and let $m^{\mathcal{S}}\left[G_{0}\right]=m^{\mathcal{B}^{c}}\left[G_{0}\right]$ be the monomial corresponding to it. Then for any reduced form $P_{n}^{\mathcal{B}^{c}}$ of $m^{\mathcal{S}}\left[G_{0}\right]$ in $\mathcal{B}^{c}\left(C_{n}\right)$, the value of $P_{n}^{\mathcal{B}^{c}}\left(x_{i j}=y_{i j}=z_{i}=1\right)$ is independent of the order of reductions performed.

\section{Reductions in the nOnCOMmutative CASE}

In this section we turn our attention to the noncommutative algebra $\mathcal{B}^{n c}\left(C_{n}\right)$. We consider reduced forms of monomials in $\mathcal{B}^{n c}\left(C_{n}\right)$, and the reduction rules correspond to the relations $(5)-\left(9^{\prime}\right)$ of $\mathcal{B}^{n c}\left(C_{n}\right)$ :

(5) $x_{i j} x_{j k} \rightarrow x_{i k} x_{i j}+x_{j k} x_{i k}$, for $1 \leq i<j<k \leq n$,

(5') $x_{j k} x_{i j} \rightarrow x_{i j} x_{i k}+x_{i k} x_{j k}$, for $1 \leq i<j<k \leq n$,

(6) $x_{i j} y_{j k} \rightarrow y_{i k} x_{i j}+y_{j k} y_{i k}$, for $1 \leq i<j<k \leq n$,

(6') $y_{j k} x_{i j} \rightarrow x_{i j} y_{i k}+y_{i k} y_{j k}$, for $1 \leq i<j<k \leq n$,

(7) $x_{i k} y_{j k} \rightarrow y_{j k} y_{i j}+y_{i j} x_{i k}$, for $1 \leq i<j<k \leq n$,

( $\left.7^{\prime}\right) y_{j k} x_{i k} \rightarrow y_{i j} y_{j k}+x_{i k} y_{i j}$, for $1 \leq i<j<k \leq n$,

(8) $y_{i k} x_{j k} \rightarrow x_{j k} y_{i j}+y_{i j} y_{i k}$, for $1 \leq i<j<k \leq n$,

(8') $x_{j k} y_{i k} \rightarrow y_{i j} x_{j k}+y_{i k} y_{i j}$, for $1 \leq i<j<k \leq n$,

(9) $x_{i j} z_{j} \rightarrow z_{i} x_{i j}+y_{i j} z_{i}+z_{j} y_{i j}$, for $i<j$,

$\left(9^{\prime}\right) z_{j} x_{i j} \rightarrow x_{i j} z_{i}+z_{i} y_{i j}+y_{i j} z_{j}$, for $i<j$.

As observed in Proposition 14] in the commutative counterpart of $\mathcal{B}^{n c}\left(C_{n}\right)$, namely $\mathcal{B}^{c}\left(C_{n}\right)$, the number of monomials in a reduced form of $w_{C_{n}}$ is the same, regardless of the order of the reductions performed. In this section we develop the tools necessary for proving the uniqueness of the reduced form in $\mathcal{B}^{n c}\left(C_{n}\right)$ for $w_{C_{n}}$ and other monomials. We introduce the concept of a "good" graph, which property is preserved under the reductions.

As in the commutative case before, we can phrase the reduction process in terms of graphs. Let $m=\prod_{l=1}^{p} \mathrm{w}\left(i_{l}, j_{l}, \epsilon_{l}\right)$ be a monomial in the variables $x_{i j}, y_{i j}, z_{k}, 1 \leq$ $i<j \leq n, k \in[n]$, where $\mathrm{w}(i, j,-)=x_{i j}$ for $i<j, \mathrm{w}(i, j,-)=x_{j i}$ for $i>j$, $\mathrm{w}(i, j,+)=y_{i j}$ and $\mathrm{w}(i, i,+)=z_{i}$. We can think of $m$ as a graph $G$ on the vertex set $[n]$ with $p$ edges labeled $1, \ldots, p$, such that the edge labeled $l$ is $\left(i_{l}, j_{l}, \epsilon_{l}\right)$. Let $G^{\mathcal{B}}[m]$ denote the edge-labeled graph just described. Let $(i, j, \epsilon)_{a}$ denote an edge $(i, j, \epsilon)$ labeled $a$. Recall that in our edge notation, $(i, j, \epsilon)=(j, i, \epsilon)$, i.e., vertex-label $i$ might be less than or greater than $j$. We can reverse the process and obtain a monomial from an edge-labeled graph $G$. Namely, if $G$ is edge-labeled with labels $1, \ldots, p$, we can also associate to it the noncommutative monomial $m^{\mathcal{B}}[G]=\prod_{a=1}^{p} \mathrm{w}\left(i_{a}, j_{a}, \epsilon_{a}\right)$, where $E(G)=\left\{\left(i_{a}, j_{a}, \epsilon_{a}\right)_{a} \mid a \in[p]\right\}$.

In terms of graphs, the partial commutativity of $\mathcal{B}^{n c}\left(C_{n}\right)$, as described by relations (2)-(4), means that if $G$ contains two edges $\left(i, j, \epsilon_{1}\right)_{a}$ and $\left(k, l, \epsilon_{2}\right)_{a+1}$ with $i, j, k, l$ distinct, then we can replace these edges by $\left(i, j, \epsilon_{1}\right)_{a+1}$ and $\left(k, l, \epsilon_{2}\right)_{a}$, and vice versa. For illustrative purposes we write out the graph reduction for relation (5) of $\mathcal{B}^{n c}\left(C_{n}\right)$. If there are two edges $(i, j,-)_{a}$ and $(j, k,-)_{a+1}$ in $G_{0}, i<j<k$, 
then we replace $G_{0}$ with two graphs $G_{1}, G_{2}$ on the vertex set $[n]$ and edge sets

$$
\begin{aligned}
& E\left(G_{1}\right)=E\left(G_{0}\right) \backslash\left\{(i, j,-)_{a}\right\} \backslash\left\{(j, k,-)_{a+1}\right\} \cup\left\{(i, k,-)_{a}\right\} \cup\left\{(i, j,-)_{a+1}\right\} \\
& E\left(G_{2}\right)=E\left(G_{0}\right) \backslash\left\{(i, j,-)_{a}\right\} \backslash\left\{(j, k,-)_{a+1}\right\} \cup\left\{(j, k,-)_{a}\right\} \cup\left\{(i, k,-)_{a+1}\right\} .
\end{aligned}
$$

Relations $\left(5^{\prime}\right)-\left(9^{\prime}\right)$ of $\mathcal{B}^{n c}\left(C_{n}\right)$ can be translated into graph language analogously. We say that $G_{0}$ reduces to $G_{1}$ and $G_{2}$ under reductions (5)- $\left(9^{\prime}\right)$.

While in the commutative case reductions on $G^{\mathcal{S}}[\mathrm{m}]$ could result in crossing graphs, we prove that in $\mathcal{B}^{n c}\left(C_{n}\right)$ all reductions preserve the noncrossing nature of the graphs, provided that we started with a suitable noncrossing graph $G$. A graph $G$ is noncrossing if there are no vertices $i<j<k<l$ and signs $\epsilon_{1}, \epsilon_{2}$ such that $\left(i, k, \epsilon_{1}\right)$ and $\left(j, l, \epsilon_{2}\right)$ are edges of $G$. We also show that under reasonable circumstances, if in $\mathcal{B}^{c}\left(C_{n}\right)$ a reduction could be applied to edges $e_{1}$ and $e_{2}$, then after suitably many allowed commutations in $\mathcal{B}^{n c}\left(C_{n}\right)$ it is possible to perform a reduction on $e_{1}$ and $e_{2}$ in $\mathcal{B}^{n c}\left(C_{n}\right)$.

We now define two central notions of the noncommutative case, that of a wellstructured graph and that of a well-labeled graph.

A graph $H$ on the vertex set $[n]$ is well-structured if it satisfies the following conditions:

(i) $H$ is noncrossing.

(ii) For any two edges $(i, j,+),(k, l,+) \in H, i<j, k<l$, it must be that $i<l$ and $k<j$.

(iii) For any two edges $(i, i,+),(k, l,+) \in H, k<l$, it must be that $k \leq i \leq l$.

(iv) There are no edges $(i, i,+),(k, j,-) \in H$ with $k<i<j$.

(v) There are no edges $(i, j,+),(k, l,-) \in H$ with $k \leq i<j \leq l$.

(vi) Graph $H$ is connected, contains exactly one loop, and contains no nonloop cycles.

Note that all positive edges in a well-structured graph are nested, and that a positive edge is never nested under a negative edge.

Condition (vi) implies that any well-structured graph on the vertex set $[n]$ contains $n$ edges.

A graph $H$ on the vertex set $[n]$ with $p$ edges labeled $1, \ldots, p$ is well-labeled if it satisfies the following conditions:

(i) If edges $\left(i, j, \epsilon_{1}\right)_{a}$ and $\left(j, k, \epsilon_{2}\right)_{b}$ are in $H, i<j<k, \epsilon_{1}, \epsilon_{2} \in\{-,+\}$, then $a<b$.

(ii) If edges $\left(i, j, \epsilon_{1}\right)_{a}$ and $\left(i, k, \epsilon_{2}\right)_{b}$ in $H$ are such that $i<j<k, \epsilon_{1}, \epsilon_{2} \in\{-,+\}$, then $a>b$.

(iii) If edges $\left(i, j, \epsilon_{1}\right)_{a}$ and $\left(k, j, \epsilon_{2}\right)_{b}$ in $H$ are such that $i<k<j, \epsilon_{1}, \epsilon_{2} \in\{-,+\}$, then $a>b$.

(iv) If edges $(i, i,+)_{a}$ and $(i, j,-)_{b}$ in $H$ are such that $i<j$, then $a<b$.

(v) If edges $(j, j,+)_{a}$ and $(i, j,-)_{b}$ in $H$ are such that $i<j$, then $a>b$.

(vi) If edges $(i, i,+)_{a}$ and $(i, j,+)_{b}$ in $H$ are such that $i<j$, then $a>b$.

(vii) If edges $(j, j,+)_{a}$ and $(i, j,+)_{b}$ in $H$ are such that $i<j$, then $a<b$.

Note that no graph $H$ with a nonloop cycle can be well-labeled. However, every well-structured graph can be well-labeled. We call graphs that are both wellstructured and well-labeled good graphs.

A $\mathcal{B}$-reduction tree $\mathcal{T}^{\mathcal{B}}$ is defined analogously to an $\mathcal{S}$-reduction tree, except that we use the noncommutative reductions to describe the children. See Figure 
8 for an example. A graph $H$ is called a $\mathcal{B}$-successor of $G$ if it is obtained by a series of reductions from $G$.

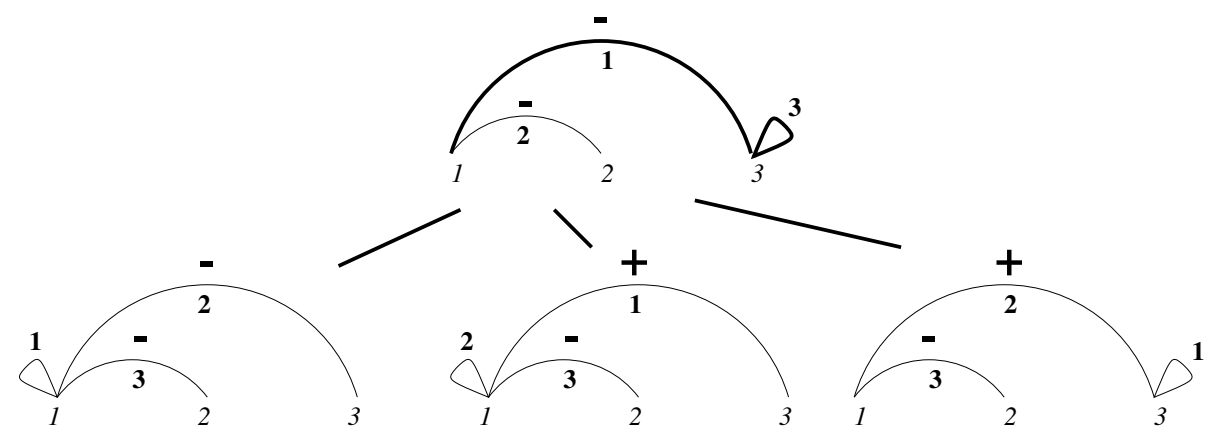

Figure 8. A $\mathcal{B}$-reduction tree with root corresponding to the monomial $x_{13} x_{12} z_{3}$. Note that in order to perform reduction on this monomial we commute the variables $x_{13}$ and $x_{12}$. In the $\mathcal{B}$ reduction tree we only record the reductions, not the commutations. Summing the monomials corresponding to the graphs labeling the leaves of the reduction tree we obtain a reduced form $P_{n}^{\mathcal{B}}$ of $x_{13} x_{12} z_{3}, P_{n}^{\mathcal{B}}=z_{1} x_{13} x_{12}+y_{13} z_{1} x_{12}+z_{3} y_{13} x_{12}$.

Lemma 15. If the root of a $\mathcal{B}$-reduction tree is labeled by a good graph, then all nodes of it are also labeled by good graphs.

The proof of Lemma 15 is an analysis of the local changes that happen during the noncommutative reduction process. An analogous lemma for type $A_{n}$ is proved in [M], Lemma 12].

A reduction applied to a noncrossing graph $G$ is noncrossing if the graphs resulting from the reduction are also noncrossing.

The following is then an immediate corollary of Lemma 15.

Corollary 16. If $G$ is a good graph, then all reductions that can be applied to $G$ and its $\mathcal{B}$-successors are noncrossing.

Let $e_{1}=\left(i_{1}, j_{1}, \epsilon_{1}\right)_{a_{1}}, e_{2}=\left(i_{2}, j_{2}, \epsilon_{2}\right)_{a_{2}}, e_{3}=\left(i_{3}, j_{3}, \epsilon_{3}\right)_{a_{3}}$ be edges of the graph $H$ such that in the commutative algebra $\mathcal{B}^{c}\left(C_{n}\right)$ a reduction could be performed on $e_{1}$ and $e_{2}$ as well as on $e_{1}$ and $e_{3}$. Suppose that $a_{1}<a_{2}<a_{3}$. Then we say, in the noncommutative case $\mathcal{B}^{n c}\left(C_{n}\right)$, that performing reduction on edges $e_{1}$ and $e_{2}$ is a priority over performing reduction on edges $e_{1}$ and $e_{3}$. We give a few concrete examples of this priority below.

Example. Performing reduction (6) on edges $(i, j,-),(j, k,+) \in H, i<j<k$, is a priority over performing reduction (9) on edges $(i, j,-),(j, j,+) \in H$. Performing reduction (9) on edges $(i, j,-),(j, j,+) \in H$ is a priority over performing reduction (5) on edges $(i, j,-),(j, k,-) \in H, i<j<k$. Performing reduction (9) on edges $(i, j,-),(j, j,+) \in H$ is a priority over performing reduction (9) on edges $(k, j,-),(j, j,+) \in H, i<k<j$. Performing reduction (9) on edges $(i, j,-),(j, j,+) \in H$ is a priority over performing reduction (8) on edges $(i, j,-),(k, j,+) \in H, k<i<j$. 
Lemma 17. Let $G$ be a good graph. Let $e_{1}$ and $e_{2}$ be edges of $G$ such that one of the reductions (5)-(9') could be applied to them in the commutative case, and such that the reduction would be noncrossing. Then after finitely many applications of allowed commutations in $\mathcal{B}^{n c}\left(C_{n}\right)$ we can perform a reduction on the edges $e_{1}$ and $e_{2}$, provided there is no edge $e_{3}$ in the graph such that reducing $e_{1}$ and $e_{3}$ or $e_{2}$ and $e_{3}$ is a priority over reducing $e_{1}$ and $e_{2}$.

The proof of Lemma 17 proceeds by inspection. An analogous lemma for type $A_{n}$ is proved in [M, Lemma 14].

\section{The Proof of Kirillov's CONJECture}

In this section we prove Conjecture 1 , construct a triangulation of $\mathcal{P}\left(C_{n}^{+}\right)$and compute its volume. In order to do this we study alternating well-structured graphs. Recall that an alternating well-structured graph $T^{l}$ is the union of a noncrossing alternating tree $T$ on the vertex set $[n]$ and a loop; that is, $T^{l}=([n], E(T) \cup$ $\{(k, k,+)\})$, for some $k \in[n]$ for which $T^{l}$ is alternating. A well-labeling that will play a special role in this section is the lexicographic labeling, defined below.

The lexicographic order on the edges of a graph $G$ with $m$ edges is as follows. Edge $\left(i_{1}, j_{1}, \epsilon\right)$ is less than edge $\left(i_{2}, j_{2}, \epsilon\right), \epsilon \in\{+,-\}$, in the lexicographic order if $j_{1}>j_{2}$, or $j_{1}=j_{2}$ and $i_{1}>i_{2}$. Furthermore, any positive edges are less than any negative edges in the lexicographic ordering. Graph $G$ is said to have lexicographic edge-labels if its edges are labeled by integers $1, \ldots, m$ such that if edge $\left(i_{1}, j_{1}, \epsilon_{1}\right)$ is less than edge $\left(i_{2}, j_{2}, \epsilon_{2}\right)$ in the lexicographic order, then the label of $\left(i_{1}, j_{1}, \epsilon_{1}\right)$ is less than the label of $\left(i_{2}, j_{2}, \epsilon_{2}\right)$ in the usual order on the integers. Given any graph $G$ there is a unique edge-labeling of it which is lexicographic. Note that our definition of lexicographic is closely related to the conventional definition, but it is not the same. For an example of lexicographic edge-labels, see the graphs labeling the leaves of the $\mathcal{B}$-reduction tree in Figure 8

Lemma 18. If $T^{l}$ is an alternating good graph, then upon some number of commutations performed on $T^{l}$, it is possible to obtain $T_{1}^{l}$ with lexicographic edge-labels.

Proof. If edges $e_{1}$ and $e_{2}$ of $T^{l}$ share a vertex and if $e_{1}$ is less than $e_{2}$ in the lexicographic order, then the label of $e_{1}$ is less than the label of $e_{2}$ in the usual order on integers by the definition of well-labeling on alternating well-structured graphs. Since commutation swaps the labels of two vertex disjoint edges labeled by consecutive integers in a graph, these swaps do not affect the relative order of the labels on edges sharing vertices. Continue these swaps until the lexicographic order is obtained.

Proposition 19. By choosing the series of reductions suitably, the set of leaves of a $\mathcal{B}$-reduction tree with root labeled by $G^{\mathcal{B}}\left[w_{C_{n}}\right]$ can all be alternating well-structured graphs $T^{l}$ on the vertex set $[n]$ with lexicographic edge-labels. The number of such graphs is $\left(\begin{array}{c}2 n-1 \\ n\end{array}\right)$.

Proof. By the correspondence between the leaves of a $\mathcal{B}$-reduction tree and simplices in a subdivision of $\mathcal{P}\left(G^{\mathcal{B}}\left[w_{C_{n}}\right]\right)$ obtained from the Reduction Lemma (Lemma 3), it follows that no graph with edge labels disregarded appears more than once among the leaves of a $\mathcal{B}$-reduction tree. Thus, it suffices to prove that any alternating wellstructured graph $T^{l}$ on the vertex set $[n]$ appears among the leaves of a $\mathcal{B}$-reduction tree and that all these graphs have lexicographic edge-labels. 
First perform all possible reductions on the graph and its successors not involving the loop $(n, n,+)$. According to [M, Theorem 18] the outcome is all noncrossing alternating spanning trees with lexicographic ordering on the vertex set $[n]$ and edge $(1, n,-)$ present. Let $T_{1}, \ldots, T_{w}$ be the trees just described and $T_{i}^{l}=\left([n], E\left(T_{i}\right) \cup\right.$ $\{(n, n,+)\}), i \in[w]$. It is clear from the definition of reductions that the only edges involved in further reducing $T_{i}^{l}, i \in[w]$ are the ones incident to vertex $n$. Thus, in order to understand what the leaves of a reduction tree with root labeled $T_{i}^{l}$, $i \in[w]$, are, it suffices to understand the leaves of a reduction tree with root labeled $G=([k+1],\{(k+1, k+1,+),(i, k+1,-) \mid i \in[k]), k \in\{1,2, \ldots, n-1\}$. It follows by inspection that the leaves of a reduction tree with root labeled $G$ are of the form $\left([k+1], E\left(G_{1}\right) \cup E\left(G_{2}\right)\right)$, where $G_{1}$ is a connected well-structured graph with only positive edges (having exactly one loop) on $[l], l \in[k+1]$, of which there are $2^{l-1}$ and $G_{2}=([k+1],\{(i, k+1) \mid i \in\{l, l+1, \ldots, k\})$. It follows that all alternating well-structured graphs $T^{l}$ are among the leaves of the particular $\mathcal{B}$-reduction tree described. Since all these graphs are well-labeled, having started with a good graph, by Lemma 18 we can assume they have lexicographic edge-labels.

From the description of the reductions above it is clear that the number of leaves of this particular reduction tree is

$$
\sum_{k=1}^{n-1} T(n, k) \cdot\left(2^{k+1}-1\right)
$$

where

$$
T(n, k)=\left(\begin{array}{c}
2 n-k-3 \\
n-k-1
\end{array}\right) \frac{k}{n-1}
$$

is the number of noncrossing alternating trees on the vertex set $[n]$ with exactly $k$ edges incident to $n$, and $2^{k+1}-1$ is the number of leaves of the reduction tree with root labeled $G([k+1],\{(k+1, k+1,+),(i, k+1,-) \mid i \in[k])$ as above. The formula for $T(n, k)$ follows by a simple bijection between noncrossing alternating trees on the vertex set $[n]$ with exactly $k$ edges incident to $n$ and ordered trees on the vertex set $[n]$ with the root having degree $k$. By equations (6.21), (6.22), (6.28) and the bijection presented in Appendix E.1 in [D], ordered trees on the vertex set $[n]$ with the root having degree $k$ are enumerated by $T(n, k)$. Since $\sum_{k=1}^{n-1} T(n, k) \cdot\left(2^{k+1}-1\right)=\left(\begin{array}{c}2 n-1 \\ n\end{array}\right)$, the proof is complete.

Theorem 20. The set of leaves of a $\mathcal{B}$-reduction tree with root labeled by $G^{\mathcal{B}}\left[w_{C_{n}}\right]$ is, up to commutations, the set of all alternating well-structured graphs on the vertex set $[n]$ with lexicographic edge-labels.

Proof. By Proposition 19 there exists a $\mathcal{B}$-reduction tree which satisfies the conditions above. By Proposition 12 the number of graphs with $n$ of the edges among the leaves of an $\mathcal{S}$-reduction tree is independent of the particular $\mathcal{S}$-reduction tree, and, thus, the same is true for a $\mathcal{B}$-reduction tree. Since all graphs labeling the leaves of a $\mathcal{B}$-reduction tree with root labeled by $G^{\mathcal{B}}\left[w_{C_{n}}\right]$ have to be good by Lemma 15, and no graph, with edge-labels disregarded, can appear twice among the leaves of a $\mathcal{B}$-reduction tree, this implies, together with Lemma 18, the statement of Theorem 20.

As corollaries of Theorem 20 we obtain the characterization of reduced forms of the noncommutative monomial $w_{C_{n}}$, a triangulation of $\mathcal{P}\left(C_{n}^{+}\right)$and a way to compute its volume. 
Theorem 21. If the polynomial $P_{n}^{\mathcal{B}}\left(x_{i j}, y_{i j}, z_{i}\right)$ is a reduced form of $w_{C_{n}}$, then up to commutations,

$$
P_{n}^{\mathcal{B}}\left(x_{i j}, y_{i j}, z_{i}\right)=\sum_{T^{l}} m^{\mathcal{B}}\left[T^{l}\right]
$$

where the sum runs over all alternating well-structured graphs $T^{l}$ on the vertex set [n] with lexicographic edge-labels.

Theorem 22. If the polynomial $P_{n}^{\mathcal{B}^{c}}\left(x_{i j}, y_{i j}, z_{i}\right)$ is a reduced form of $w_{C_{n}}$ in $\mathcal{B}^{c}\left(C_{n}\right)$, then

$$
P_{n}^{\mathcal{B}^{c}}\left(x_{i j}=y_{i j}=z_{i}=1\right)=\left(\begin{array}{c}
2 n-1 \\
n
\end{array}\right) .
$$

Proof. Proposition 14 and Theorem 21 imply $P_{n}^{\mathcal{B}^{c}}\left(x_{i j}=y_{i j}=z_{i}=1\right)=\left(\begin{array}{c}2 n-1 \\ n\end{array}\right)$.

Theorem 23. Let $T_{1}^{l}, \ldots, T_{m}^{l}$ be all alternating well-structured graphs on the vertex set $[n]$. Then $\mathcal{P}\left(T_{1}^{l}\right), \ldots, \mathcal{P}\left(T_{m}^{l}\right)$ are $n$-dimensional simplices forming a triangulation of $\mathcal{P}\left(C_{n}^{+}\right)$. Furthermore,

$$
\operatorname{vol}_{n}\left(\mathcal{P}\left(C_{n}^{+}\right)\right)=\left(\begin{array}{c}
2 n-1 \\
n
\end{array}\right) \frac{2}{n !} .
$$

Proof. The Reduction Lemma implies the first claim, and Proposition 10 implies that $\operatorname{vol}_{n}\left(\mathcal{P}\left(C_{n}^{+}\right)\right)=\left(\begin{array}{c}2 n-1 \\ n\end{array}\right) \frac{2}{n !}$.

The value of the volume of $\mathcal{P}\left(C_{n}^{+}\right)$has previously been observed by Fong [F. p. 55].

\section{The General CASE}

In this section we find analogues of Theorems 20, 21, 22, and 23 for any wellstructured graph $T^{l}$ on the vertex set $[n]$.

Proposition 24. Let $T^{l}$ be a well-structured graph on the vertex set $[n]$. By choosing the series of reductions suitably, the set of leaves of a $\mathcal{B}$-reduction tree with root labeled by $T^{l}$ can all be alternating well-structured spanning graphs $G$ of $\overline{T^{l}}$ on the vertex set $[n]$ with lexicographic edge-labels.

Proof. All graphs labeling the leaves of a $\mathcal{B}$-reduction tree must be alternating well-structured spanning graphs $G$ of $\overline{T^{l}}$. Also, it is possible to obtain any wellstructured graph $T^{l}$ on the vertex set $[n]$ as a $\mathcal{B}$-successor of $P^{l}$. Furthermore, if $T^{l}$ and $T_{1}^{l}$ are two $\mathcal{B}$-successors of $P^{l}$ in the same $\mathcal{B}$-reduction tree, and neither is the $\mathcal{B}$-successor of the other, then the intersection of $\overline{T^{l}}$ and $\overline{T_{1}^{l}}$ does not contain a well-structured graph $G$, as the existence of such a graph would imply that $\mathcal{P}\left(T^{l}\right)$ and $\mathcal{P}\left(T_{1}^{l}\right)$ have a common interior point, contrary to the Reduction Lemma. Since the set of leaves of a $\mathcal{B}$-reduction tree with root labeled by $P^{l}$ is, up to commutations, the set of all alternating well-structured graphs on the vertex set $[n]$ with lexicographic edge-labels according to Theorem 20. Proposition 24 follows.

Theorem 25. Let $T^{l}$ be a well-structured graph on the vertex set $[n]$. The set of leaves of a $\mathcal{B}$-reduction tree with root labeled $T^{l}$ is, up to commutations, the set of all alternating well-structured spanning graphs $G$ of $\overline{T^{l}}$ on the vertex set $[n]$ with lexicographic edge-labels. 
Proof. The proof is analogous to that of Theorem 20 using Proposition 24 instead of Proposition 19 .

As corollaries of Theorem 25 we obtain the characterization of reduced forms of the noncommutative monomial $m^{\mathcal{B}}\left[T^{l}\right]$, a triangulation of $\mathcal{P}\left(T^{l}\right)$ and a way to compute its volume, for a well-structured graph $T^{l}$ on the vertex set $[n]$.

Theorem 26 (Noncommutative part). If the polynomial $P_{n}^{\mathcal{B}}\left(x_{i j}, y_{i j}, z_{i}\right)$ is a reduced form of $m^{\mathcal{B}}\left[T^{l}\right]$ for a well-structured graph $T^{l}$ on the vertex set $[n]$, then up to commutations,

$$
P_{n}^{\mathcal{B}}\left(x_{i j}, y_{i j}, z_{i}\right)=\sum_{G} m^{\mathcal{B}}[G]
$$

where the sum runs over all alternating well-structured spanning graphs $G$ of $\overline{T^{l}}$ on the vertex set $[n]$ with lexicographic edge-labels.

Theorem 27 (Commutative part). If the polynomial $P_{n}^{\mathcal{B}^{c}}\left(x_{i j}, y_{i j}, z_{i}\right)$ is a reduced form of $m^{\mathcal{B}^{c}}\left[T^{l}\right]$ for a well-structured graph $T^{l}$ on the vertex set $[n]$, then

$$
P_{n}^{\mathcal{B}^{c}}\left(x_{i j}=y_{i j}=z_{i}=1\right)=f_{T^{l}},
$$

where $f_{T^{l}}$ is the number of alternating well-structured spanning graphs $G$ of $\overline{T^{l}}$.

Theorem 28 (Triangulation and volume). Let $T_{1}^{l}, \ldots, T_{m}^{l}$ be all alternating wellstructured spanning graphs of $\overline{T^{l}}$ for a well-structured graph $T^{l}$ on the vertex set $[n]$. Then $\mathcal{P}\left(T_{1}^{l}\right), \ldots, \mathcal{P}\left(T_{m}^{l}\right)$ are $n$-dimensional simplices forming a triangulation of $\mathcal{P}\left(T^{l}\right)$. Furthermore,

$$
\operatorname{vol}_{n}\left(\mathcal{P}\left(T^{l}\right)\right)=f_{T^{l}} \frac{2}{n !},
$$

where $f_{T^{l}}$ is the number of alternating well-structured spanning graphs $G$ of $\overline{T^{l}}$.

\section{A more general noncommutative Algebra $\mathcal{B}^{\beta}\left(C_{n}\right)$}

In this section we define the noncommutative algebra $\mathcal{B}^{\beta}\left(C_{n}\right)$, which specializes to $\mathcal{B}^{n c}\left(C_{n}\right)$ when we set $\beta=0$. We prove analogs of the results presented so far for this more general algebra. We also provide a way for calculating Ehrhart polynomials for certain type $C_{n}$ root polytopes.

Let the $\beta$-bracket algebra $\mathcal{B}^{\beta}\left(C_{n}\right)$ of type $C_{n}$ be an associative algebra over $\mathbb{Q}[\beta]$, where $\beta$ is a variable (and a central element), with a set of generators $\left\{x_{i j}, y_{i j}, z_{i} \mid 1 \leq i \neq j \leq n\right\}$ subject to the following relations:

(1) $x_{i j}+x_{j i}=0, y_{i j}=y_{j i}$, for $i \neq j$,

(2) $z_{i} z_{j}=z_{j} z_{i}$

(3) $x_{i j} x_{k l}=x_{k l} x_{i j}, y_{i j} x_{k l}=x_{k l} y_{i j}, y_{i j} y_{k l}=y_{k l} y_{i j}$, for $i<j, k<l$ distinct,

(4) $z_{i} x_{k l}=x_{k l} z_{i}, z_{i} y_{k l}=y_{k l} z_{i}$, for all $i \neq k, l$,

(5) $x_{i j} x_{j k}=x_{i k} x_{i j}+x_{j k} x_{i k}+\beta x_{i k}$, for $1 \leq i<j<k \leq n$,

(5') $x_{j k} x_{i j}=x_{i j} x_{i k}+x_{i k} x_{j k}+\beta x_{i k}$, for $1 \leq i<j<k \leq n$,

(6) $x_{i j} y_{j k}=y_{i k} x_{i j}+y_{j k} y_{i k}+\beta y_{i k}$, for $1 \leq i<j<k \leq n$,

(6') $y_{j k} x_{i j}=x_{i j} y_{i k}+y_{i k} y_{j k}+\beta y_{i k}$, for $1 \leq i<j<k \leq n$,

(7) $x_{i k} y_{j k}=y_{j k} y_{i j}+y_{i j} x_{i k}+\beta y_{i j}$, for $1 \leq i<j<k \leq n$,

(7') $y_{j k} x_{i k}=y_{i j} y_{j k}+x_{i k} y_{i j}+\beta y_{i j}$, for $1 \leq i<j<k \leq n$,

(8) $y_{i k} x_{j k}=x_{j k} y_{i j}+y_{i j} y_{i k}+\beta y_{i j}$, for $1 \leq i<j<k \leq n$,

$\left(8^{\prime}\right) x_{j k} y_{i k}=y_{i j} x_{j k}+y_{i k} y_{i j}+\beta y_{i j}$, for $1 \leq i<j<k \leq n$, 
(9) $x_{i j} z_{j}=z_{i} x_{i j}+y_{i j} z_{i}+z_{j} y_{i j}+\beta z_{i}+\beta y_{i j}$, for $1 \leq i<j \leq n$,

$\left(9^{\prime}\right) z_{j} x_{i j}=x_{i j} z_{i}+z_{i} y_{i j}+y_{i j} z_{j}+\beta z_{i}+\beta y_{i j}$, for $1 \leq i<j \leq n$.

Kirillov [K2] made Conjecture 1 not just for $\mathcal{B}^{n c}\left(C_{n}\right)$, but for a more general $\beta$-bracket algebra of type $C_{n}$, which is almost identical to $\mathcal{B}^{\beta}\left(C_{n}\right)$; it differs in a term in relations $(9)$ and $\left(9^{\prime}\right)$. We prove the analogue of Conjecture 1 for $\mathcal{B}^{\beta}\left(C_{n}\right)$.

Notice that the commutativization of $\mathcal{B}^{\beta}\left(C_{n}\right)$ yields the relations of $\mathcal{S}\left(C_{n}\right)$, except for relations $(9)$ and $\left(9^{\prime}\right)$ of $\mathcal{B}^{\beta}\left(C_{n}\right)$, which can be obtained by combining relations (6) and (7) of $\mathcal{S}\left(C_{n}\right)$. Since the Reduction Lemma (Lemma 3) holds for $\mathcal{S}\left(C_{n}\right)$, so does it for $\mathcal{B}^{\beta}\left(C_{n}\right)$, keeping in mind that relations $(9)$ and $\left(9^{\prime}\right)$ of $\mathcal{B}^{\beta}\left(C_{n}\right)$ are obtained by combining relations (6) and (7) of $\mathcal{S}\left(C_{n}\right)$. As a result, we can think of relations (5)- $\left(9^{\prime}\right)$ of $\mathcal{B}^{\beta}\left(C_{n}\right)$ as operations subdividing root polytopes into smaller polytopes and keeping track of their lower-dimensional intersections.

A $\mathcal{B}^{\beta}$-reduction tree is analogous to an $\mathcal{S}$-reduction tree, just that the children of the nodes are obtained by the relations $(5)-\left(9^{\prime}\right)$ of $\mathcal{B}^{\beta}\left(C_{n}\right)$, and now some nodes have five, and some nodes have three children. See Figure 9 for an example. If $\mathcal{T}^{\mathcal{B}^{\beta}}$ is a $\mathcal{B}^{\beta}$-reduction tree with root labeled $G$ and leaves labeled by the graphs $G_{1}, \ldots, G_{q}$, then

$$
\mathcal{P}^{\circ}(G)=\mathcal{P}^{\circ}\left(G_{1}\right) \cup \cdots \cup \mathcal{P}^{\circ}\left(G_{q}\right),
$$

by an analogue of the Reduction Lemma.

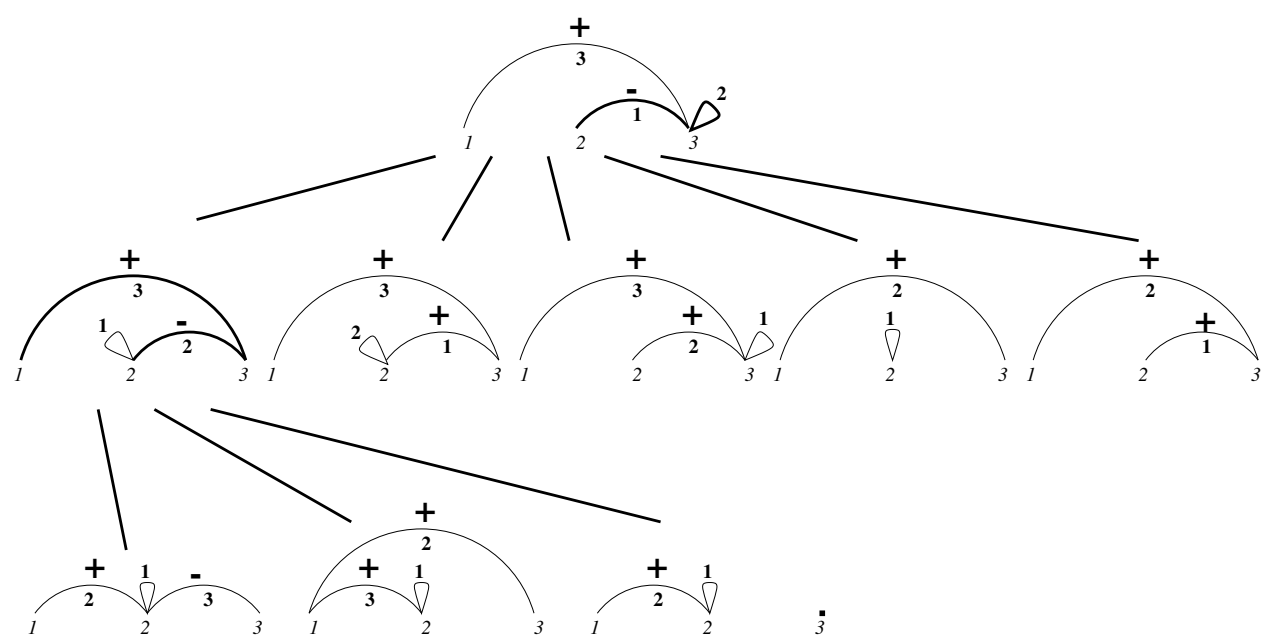

Figure 9. A $\mathcal{B}^{\beta}$-reduction tree with root corresponding to the monomial $x_{23} z_{3} y_{13}$. Summing the monomials corresponding to the graphs labeling the leaves of the reduction tree multiplied by suitable powers of $\beta$, we obtain a reduced form $P_{n}^{\mathcal{B}^{\beta}}$ of $x_{23} z_{3} y_{13}$, $P_{n}^{\mathcal{B}^{\beta}}=z_{2} y_{12} x_{23}+z_{2} y_{13} y_{12}+\beta z_{2} y_{12}+y_{23} z_{2} y_{13}+z_{3} y_{23} y_{13}+\beta z_{2} y_{13}$ $+\beta y_{23} y_{13}$.

In order to prove an analogue of Proposition 19 for the algebra $\mathcal{B}^{\beta}\left(C_{n}\right)$, we need a definition more general than well-structured. Thus we now define weakly-wellstructured graphs. 
A graph $H$ on the vertex set $[n]$ with $p \leq n$ edges is weakly-well-structured if it satisfies the following conditions:

(i) $H$ is noncrossing.

(ii) For any two edges $(i, j,+),(k, l,+) \in H, i<j, k<l$, it must be that $i<l$ and $k<j$.

(iii) For any two edges $(i, i,+),(k, l,+) \in H, k<l$, it must be that $k \leq i \leq l$.

(iv) There are no edges $(i, i,+),(k, j,-) \in H$ with $k<i<j$.

(v) There are no edges $(i, j,+),(k, l,-) \in H$ with $k \leq i<j \leq l$.

(vi) Graph $H$ contains at most one loop, and $H$ contains no nonloop cycles.

(vii) Graph $H$ contains a positive edge incident to vertex 1.

Note that well-structured graphs are also weakly-well-structured.

Proposition 29. By choosing the set of reductions suitably, the set of leaves of a $\mathcal{B}^{\beta}$-reduction tree $\mathcal{T}^{\mathcal{B}^{\beta}}$ with root labeled by $P^{l}=([n],\{(n, n,+),(i, i+1,-) \mid i \in$ $[n-1]\})$ can be the set of all alternating weakly-well-structured subgraphs $G$ of $\overline{P^{l}}$ with lexicographic edge-labels.

Proof. The proof of Proposition 29 proceeds analogously to that of Proposition 19. using equation (19), instead of the original statement of the Reduction Lemma, and using the full statement of [M, Theorem 18] which says that the leaves of a reduction tree with root labeled by $([n],\{(i, i+1,-) \mid i \in[n-1]\})$ are all noncrossing alternating forests with negative edges on the vertex set $[n]$ containing edge $(1, n,-)$ with lexicographic edge-labels.

Theorem 30. The set of leaves of a $\mathcal{B}^{\beta}$-reduction tree $\mathcal{T}^{\mathcal{B}^{\beta}}$ with root labeled $P^{l}$ is, up to commutations, the set of all alternating weakly-well-structured subgraphs $G$ of $\overline{P^{l}}$ with lexicographic edge-labels.

Proof. Proposition 29 proves the existence of one such $\mathcal{B}^{\beta}$-reduction tree. An analogue of Lemma 15 states that if the root of a $\mathcal{B}^{\beta}$-reduction tree is a weakly-wellstructured well-labeled graph, then so are all its nodes. Together with equation (19) these imply Theorem 30.

As corollaries of Theorem 30 we obtain the characterization of reduced forms of the noncommutative monomial $w_{C_{n}}$ in $\mathcal{B}^{\beta}\left(C_{n}\right)$ as well as a canonical triangulation of $\mathcal{P}\left(P^{l}\right)$ and an expression for its Ehrhart polynomial.

Theorem 31. If the polynomial $P_{n}^{\mathcal{B}^{\beta}}\left(x_{i j}, y_{i j}, z_{i}\right)$ is a reduced form of $w_{C_{n}}$ in $\mathcal{B}^{\beta}\left(C_{n}\right)$, then

$$
P_{n}^{\mathcal{B}^{\beta}}\left(x_{i j}, y_{i j}, z_{i}\right)=\sum_{G} \beta^{n-|E(G)|} m^{\mathcal{B}}[G]
$$

where the sum runs over all alternating weakly-well-structured graphs $G$ on the vertex set $[n]$ with lexicographic edge-labels.

Theorem 32 (Canonical triangulation). Let $G_{1}, \ldots, G_{k}$ be all the alternating wellstructured graphs on the vertex set $[n]$. Then the root polytopes $\mathcal{P}\left(G_{1}\right), \ldots, \mathcal{P}\left(G_{k}\right)$ are $n$-dimensional simplices forming a triangulation of $\mathcal{P}\left(P^{l}\right)$. Furthermore, the intersections of the top-dimensional simplices $\mathcal{P}\left(G_{1}\right), \ldots, \mathcal{P}\left(G_{k}\right)$ are simplices $\mathcal{P}(H)$, where $H$ runs over all alternating weakly-well-structured graphs on the vertex set $[n]$. 
Given a polytope $\mathcal{P} \subset \mathbb{R}^{n}$, the $t^{\text {th }}$ dilate of $\mathcal{P}$ is

$$
t \mathcal{P}=\left\{\left(t x_{1}, \ldots, t x_{n}\right) \mid\left(x_{1}, \ldots, x_{n}\right) \in \mathcal{P}\right\} .
$$

The Ehrhart polynomial of an integer polytope $\mathcal{P} \subset \mathbb{R}^{n}$ is

$$
L_{\mathcal{P}}(t)=\#\left(t \mathcal{P} \cap \mathbb{Z}^{n}\right)
$$

For background on the theory of Ehrhart polynomials, see [BR].

Theorem 33 (Ehrhart polynomial).

$$
\begin{aligned}
L_{\mathcal{P}\left(P^{l}\right)}(t)=(-1)^{n}( & \sum_{d=1}^{n} f^{l}(d)(-1)^{d}\left(\left(\begin{array}{c}
d+t \\
d
\end{array}\right)+\left(\begin{array}{c}
d+t-1 \\
d
\end{array}\right)\right) \\
& \left.+\sum_{d=1}^{n-1} f(d)(-1)^{d}\left(\begin{array}{c}
d+t \\
d
\end{array}\right)\right),
\end{aligned}
$$

where $f^{l}(d)$ is the number of alternating weakly-well-structured graphs on the vertex set $[n]$ with $d$ edges one of which is a loop and $f(d)$ is the number of alternating weakly-well-structured graphs on the vertex set [n] with d edges and no loops.

Proof. By Theorem 32, $\mathcal{P}\left(P^{l}\right)^{\circ}=\bigsqcup_{F \in W} \mathcal{P}(F)^{\circ} \sqcup \bigsqcup_{F^{l} \in W^{l}} \mathcal{P}\left(F^{l}\right)^{\circ}$, where $W$ is the set of all alternating weakly-well-structured graphs on the vertex set $[n]$ with no loops and $W^{l}$ is the set of all alternating weakly-well-structured graphs on the vertex set $[n]$ with a loop. Then

$$
L_{\mathcal{P}\left(P^{l}\right)^{\circ}}(t)=\sum_{F \in W} L_{\mathcal{P}(F)^{\circ}}(t)+\sum_{F^{l} \in W^{l}} L_{\mathcal{P}\left(F^{l}\right)^{\circ}}(t) .
$$

By [S1, Theorem 1.3] the Ehrhart series of $\mathcal{P}(F), F \in W, \# E(F)=d$, and $\mathcal{P}\left(F^{l}\right)$, $F^{l} \in W^{l}, \# E\left(F^{l}\right)=d$, respectively, are $J(\mathcal{P}(F), x)=1+\sum_{t=1}^{\infty} L_{\mathcal{P}(F)}(t) x^{t}=$ $\frac{1}{(1-x)^{d+1}}$ and $J\left(\mathcal{P}\left(F^{l}\right), x\right)=\frac{1+x}{(1-x)^{d+1}}$. Equivalently, $L_{\mathcal{P}(F)^{\circ}}(t)=\left(\begin{array}{c}t-1 \\ d\end{array}\right), L_{\mathcal{P}\left(F^{l}\right)^{\circ}}(t)=$ $\left(\begin{array}{c}t-1 \\ d\end{array}\right)+\left(\begin{array}{l}t \\ d\end{array}\right)$. Thus,

$$
L_{\mathcal{P}\left(P^{l}\right)^{\circ}}(t)=\sum_{d=1}^{n} f^{l}(d)\left(\left(\begin{array}{c}
t-1 \\
d
\end{array}\right)+\left(\begin{array}{l}
t \\
d
\end{array}\right)\right)+\sum_{d=1}^{n-1} f(d)\left(\begin{array}{c}
t-1 \\
d
\end{array}\right),
$$

where $f^{l}(d)=\#\left\{F^{l} \in W^{l} \mid \# E\left(F^{l}\right)=d\right\}, f(d)=\#\{F \in W \mid \# E(F)=d\}$. Using the Ehrhart-Macdonald reciprocity [BR, Theorem 4.1],

$$
\begin{aligned}
& L_{\mathcal{P}\left(P^{l}\right)}(t)=(-1)^{n} L_{\mathcal{P}\left(P^{l}\right)^{\circ}}(-t) \\
& =(-1)^{n}\left(\sum_{d=1}^{n} f^{l}(d)(-1)^{d}\left(\left(\begin{array}{c}
d+t \\
d
\end{array}\right)+\left(\begin{array}{c}
d+t-1 \\
d
\end{array}\right)\right)+\sum_{d=1}^{n-1} f(d)(-1)^{d}\left(\begin{array}{c}
d+t \\
d
\end{array}\right)\right) .
\end{aligned}
$$

Theorems 30, 31, 32 and 33 can be generalized to any well-structured graph $G$ by adding further technical requirements on the weakly-well-structured graphs that 
can appear among the leaves of a $\mathcal{B}^{\beta}$-reduction tree with root labeled by $G$. Due to the technical nature of these results, we omit them here.

\section{The TYPe $D_{n}$ BRACKET ALGEBRA}

In the rest of the paper we study the reduced forms of elements in the type $D_{n}$ bracket algebra with combinatorial methods fused with noncommutative Gröbner basis theory. While the connection with subdivisions of type $C_{n}$ root polytopes is present in this case as well, for brevity we choose to suppress this aspect.

Let the $\beta$-bracket algebra $\mathcal{B}^{\beta}\left(D_{n}\right)$ of type $D_{n}$ be an associative algebra over $\mathbb{Q}[\beta]$, where $\beta$ is a variable (and a central element), with a set of generators $\left\{x_{i j}, y_{i j} \mid\right.$ $1 \leq i \neq j \leq n\}$ subject to the following relations:

(1) $x_{i j}+x_{j i}=0, y_{i j}=y_{j i}$, for $i \neq j$,

(2) $z_{i} z_{j}=z_{j} z_{i}$,

(3) $x_{i j} x_{k l}=x_{k l} x_{i j}, y_{i j} x_{k l}=x_{k l} y_{i j}, y_{i j} y_{k l}=y_{k l} y_{i j}$, for $i<j, k<l$ distinct,

(4) $z_{i} x_{k l}=x_{k l} z_{i}, z_{i} y_{k l}=y_{k l} z_{i}$, for all $i \neq k, l$,

(5) $x_{i j} x_{j k}=x_{i k} x_{i j}+x_{j k} x_{i k}+\beta x_{i k}$, for $1 \leq i<j<k \leq n$,

(5') $x_{j k} x_{i j}=x_{i j} x_{i k}+x_{i k} x_{j k}+\beta x_{i k}$, for $1 \leq i<j<k \leq n$,

(6) $x_{i j} y_{j k}=y_{i k} x_{i j}+y_{j k} y_{i k}+\beta y_{i k}$, for $1 \leq i<j<k \leq n$,

$\left(6^{\prime}\right) y_{j k} x_{i j}=x_{i j} y_{i k}+y_{i k} y_{j k}+\beta y_{i k}$, for $1 \leq i<j<k \leq n$,

(7) $x_{i k} y_{j k}=y_{j k} y_{i j}+y_{i j} x_{i k}+\beta y_{i j}$, for $1 \leq i<j<k \leq n$,

$\left(7^{\prime}\right) y_{j k} x_{i k}=y_{i j} y_{j k}+x_{i k} y_{i j}+\beta y_{i j}$, for $1 \leq i<j<k \leq n$,

(8) $y_{i k} x_{j k}=x_{j k} y_{i j}+y_{i j} y_{i k}+\beta y_{i j}$, for $1 \leq i<j<k \leq n$,

$\left(8^{\prime}\right) x_{j k} y_{i k}=y_{i j} x_{j k}+y_{i k} y_{i j}+\beta y_{i j}$, for $1 \leq i<j<k \leq n$.

Note that $\mathcal{B}^{\beta}\left(C_{n}\right)$ is a quotient of $\mathcal{B}^{\beta}\left(D_{n}\right)$, since $\mathcal{B}^{\beta}\left(C_{n}\right)$ has all the above relations and in addition relations $(9),\left(9^{\prime}\right)$; see Section 11 .

Let $w_{D_{n}}=\prod_{i=1}^{n-1} x_{i, i+1} y_{n-1, n}$ be a Coxeter type element in $\mathcal{B}^{\beta}\left(D_{n}\right)$ and let $P_{n}^{\mathcal{B}}$ be a polynomial in variables $x_{i j}, y_{i j}, 1 \leq i \neq j \leq n$ obtained from $w_{D_{n}}$ by successively applying the defining relations $(5)-\left(8^{\prime}\right)$ in any order until unable to do so, in the algebra $\mathbb{Q}[\beta]\left\langle x_{i j}, y_{i j} \mid 1 \leq i<j \leq n\right\rangle / I$, where $I$ is the (two-sided) ideal generated by the relations (1)-(4). We call $P_{n}^{\mathcal{B}}$ a reduced form of $w_{D_{n}}$ and consider the process of successively applying the defining relations $(5)-\left(8^{\prime}\right)$ as a reduction process in $\mathbb{Q}[\beta]\left\langle x_{i j}, y_{i j} \mid 1 \leq i<j \leq n\right\rangle / I$, with the reduction rules:

(5) $x_{i j} x_{j k} \rightarrow x_{i k} x_{i j}+x_{j k} x_{i k}+\beta x_{i k}$, for $1 \leq i<j<k \leq n$,

(5') $x_{j k} x_{i j} \rightarrow x_{i j} x_{i k}+x_{i k} x_{j k}+\beta x_{i k}$, for $1 \leq i<j<k \leq n$,

(6) $x_{i j} y_{j k} \rightarrow y_{i k} x_{i j}+y_{j k} y_{i k}+\beta y_{i k}$, for $1 \leq i<j<k \leq n$,

$\left(6^{\prime}\right) y_{j k} x_{i j} \rightarrow x_{i j} y_{i k}+y_{i k} y_{j k}+\beta y_{i k}$, for $1 \leq i<j<k \leq n$,

(7) $x_{i k} y_{j k} \rightarrow y_{j k} y_{i j}+y_{i j} x_{i k}+\beta y_{i j}$, for $1 \leq i<j<k \leq n$,

$\left(7^{\prime}\right) y_{j k} x_{i k} \rightarrow y_{i j} y_{j k}+x_{i k} y_{i j}+\beta y_{i j}$, for $1 \leq i<j<k \leq n$,

(8) $y_{i k} x_{j k} \rightarrow x_{j k} y_{i j}+y_{i j} y_{i k}+\beta y_{i j}$, for $1 \leq i<j<k \leq n$,

(8') $x_{j k} y_{i k} \rightarrow y_{i j} x_{j k}+y_{i k} y_{i j}+\beta y_{i j}$, for $1 \leq i<j<k \leq n$.

The reduced form of any other element of $\mathcal{B}^{\beta}\left(D_{n}\right)$ is defined analogously. As in the type $C_{n}$ case, the relations of $\mathcal{B}^{\beta}\left(D_{n}\right)$ can be interpreted as subdividing type $C_{n}$ root polytopes and the reduced form of an element as a subdivision, though not a triangulation, of a type $C_{n}$ polytope. We pursue a different approach to studying reduced forms here.

We can think of the reduction process in $\mathbb{Q}\left\langle\beta, x_{i j}, y_{i j} \mid 1 \leq i<j \leq n\right\rangle / I_{\beta}$, where the generators of the (two-sided) ideal $I_{\beta}$ are those of $I$ and in addition the commutators of $\beta$ with all the other variables $x_{i j}, y_{i j}, 1 \leq i<j \leq n$. 
Conjecture 2 (Kirillov [K2]). Apart from applying the relations (1)-(4), the reduced form $P_{n}^{\mathcal{B}}$ of $w_{D_{n}}$ does not depend on the order in which the reductions are performed.

Note that the above statement does not hold true for any monomial; some examples illustrating this were already explained in the comments after Conjecture 1 in Section 2 ,

\section{GRAPhS FOR TYPE $D_{n}$}

It is straightforward to reformulate the reduction rules $(5)-\left(8^{\prime}\right)$ in terms of reductions on graphs. If $m \in \mathcal{B}^{\beta}\left(D_{n}\right)$, then we replace each monomial $m$ in the reductions by corresponding graphs $G^{\mathcal{B}}[\mathrm{m}]$. The analogous procedure for type $C_{n}$ is explained in detail in Section 8

We now define a central notion for those signed graphs whose corresponding monomials turn out to have a unique reduced form in $\mathcal{B}^{\beta}\left(D_{n}\right)$. We reuse the expression "good graph" from the type $C_{n}$ case, though the meaning in type $D_{n}$ is different. Previously we used good in the type $C_{n}$ sense; in the following we use good in the type $D_{n}$ sense.

A graph $H$ on the vertex set $[n]$ with $k$ edges labeled $1, \ldots, k$ is good if it satisfies the following conditions:

(i) The negative edges of $H$ form a noncrossing graph.

(ii) If edges $(i, j,-)_{a}$ and $\left(j, k, \epsilon_{2}\right)_{b}$ are in $H, i<j<k, \epsilon_{2} \in\{-,+\}$, then $a<b$.

(iii) If edges $(i, j,-)_{a}$ and $\left(i, k, \epsilon_{2}\right)_{b}$ are in $H, i<j<k, \epsilon_{2} \in\{-,+\}$, then $a>b$.

(iv) If edges $(j, k,-)_{a}$ and $\left(i, k, \epsilon_{2}\right)_{b}$ are in $H, i<j<k, \epsilon_{2} \in\{-,+\}$, then $a<b$.

(v) If edges $(j, k,+)_{a}$ and $(i, k,-)_{b}$ are in $H, i<j<k$, then $a>b$.

(vi) If edges $(i, k,-)_{a}$ and $(j, l,+)_{b}$ are in $H, i<j<k<l$, then $a>b$.

Lemma 34. If $H$ is a good graph, then reduction rules $\left(5^{\prime}\right),\left(6^{\prime}\right),\left(7^{\prime}\right),(8)$ cannot be performed on it. If we perform any of the reduction rules $(5),(6),(7),\left(8^{\prime}\right)$ on $H$, then we obtain a graph $H^{r}$, which is also a good graph.

Proof. Note that there is no way of commuting the labels of good graphs so as to obtain an order on the edges which would allow rules $\left(5^{\prime}\right),\left(6^{\prime}\right),\left(7^{\prime}\right),(8)$ to be performed.

That the following properties carry over from $H$ to $H^{r}$ follows from $\mathbb{M}$, Lemma 12], noting that only reduction rule (5) creates new negative edges:

- The negative edges of $H$ form a noncrossing graph.

- If edges $(i, j,-)_{a}$ and $(j, k,-)_{b}$ are in $H, i<j<k$, then $a<b$.

- If edges $(i, j,-)_{a}$ and $(i, k,-)_{b}$ are in $H, i<j<k$, then $a>b$.

- If edges $(j, k,-)_{a}$ and $(i, k,-)_{b}$ are in $H, i<j<k$, then $a<b$.

Inspection shows that the following properties carry over from $H$ to $H^{r}$, keeping in mind that the above properties carry over for negative edges.

- If edges $(i, j,-)_{a}$ and $(j, k,+)_{b}$ are in $H, i<j<k$, then $a<b$.

- If edges $(j, k,-)_{a}$ and $(i, k,+)_{b}$ are in $H, i<j<k$, then $a<b$.

- If edges $(j, k,+)_{a}$ and $(i, k,-)_{b}$ are in $H, i<j<k$, then $a>b$.

- If edges $(i, k,-)_{a}$ and $(j, l,+)_{b}$ are in $H, i<j<k<l$, then $a>b$. 
Finally, given that all the above properties carry over from $H$ to $H^{r}$, it follows that the property

- If edges $(i, j,-)_{a}$ and $(i, k,+)_{b}$ are in $H, i<j<k$, then $a>b$

also carries over.

Why are good graphs so good? Well, if the relations $(5),(6),(7),\left(8^{\prime}\right)$ were a noncommutative Gröbner basis for the ideal they generate in $\mathbb{Q}\left\langle\beta, x_{i j}, y_{i j}\right| 1 \leq$ $i<j \leq n\rangle / I_{\beta}$, with tips $x_{i j} x_{j k}, x_{i j} y_{j k}, x_{i k} y_{j k}, x_{j k} y_{i k}$, respectively, then it would follow immediately that the reduced form of monomials corresponding to good graphs is unique by results in noncommutative Gröbner bases theory. As it turns out, the previous situation is not the case; however, we can still use Gröbner bases to prove the uniqueness of the reduced forms of the monomials corresponding to good graphs, which we call good monomials, with a little bit more work. We show how to do this in the next section.

\section{GRÖBNER BASES}

In this section we briefly review some facts about noncommutative Gröbner bases and use them to show that the reduced forms of good monomials are unique.

We use the terminology and notation of $[\mathrm{G}$, but state the results only for our special algebra. For the more general statements, see $\mathrm{G}$. Throughout this section we consider the noncommutative case only.

Let

$$
\mathbf{R}=\mathbb{Q}\left\langle\beta, \mathbf{x}_{\mathbf{i j}}, \mathbf{y}_{\mathbf{i j}} \mid \mathbf{1} \leq \mathbf{i}<\mathbf{j} \leq \mathbf{n}\right\rangle / \mathbf{I}_{\beta}
$$

with multiplicative basis $\mathcal{B}$, the set of noncommutative monomials up to equivalence under the commutativity relations described by $I_{\beta}$.

The tip of an element $f \in R$ is the largest basis element appearing in its expansion, denoted by $\operatorname{Tip}(f)$. Let $\operatorname{CTip}(f)$ denote the coefficient of $\operatorname{Tip}(f)$ in this expansion. A set of elements $X$ is tip reduced if for distinct elements $x, y \in X$, $\operatorname{Tip}(x)$ does not divide $\operatorname{Tip}(y)$.

A well-order $>$ on $\mathcal{B}$ is admissible if for $p, q, r, s \in \mathcal{B}$ :

1. if $p<q$, then $p r<q r$ if both $p r \neq 0$ and $q r \neq 0$;

2. if $p<q$, then $s p<s q$ if both $s p \neq 0$ and $s q \neq 0$;

3. if $p=q r$, then $p>q$ and $p>r$.

Let $f, g \in R$ and suppose that there are monomials $b, c \in \mathcal{B}$ such that

1. $\operatorname{Tip}(f) c=b \operatorname{Tip}(g)$.

2. $\operatorname{Tip}(f)$ does not divide $b$ and $\operatorname{Tip}(g)$ does not divide $c$.

Then the overlap relation of $f$ and $g$ by $b$ and $c$ is

$$
o(f, g, b, c)=\frac{f c}{\operatorname{CTip}(f)}-\frac{b g}{\operatorname{CTip}(g)} .
$$

Proposition 35 ([G, Theorem 2.3]). A tip reduced generating set of elements $\mathcal{G}$ of the ideal $J$ of $R$ is a Gröbner basis, where the ordering on the monomials is admissible, if for every overlap relation,

$$
o\left(g_{1}, g_{2}, p, q\right) \Rightarrow_{\mathcal{G}} 0
$$

where $g_{1}, g_{2} \in \mathcal{G}$ and the above notation means that dividing $o\left(g_{1}, g_{2}, p, q\right)$ by $\mathcal{G}$ yields a remainder of 0 . 
See [G, Theorem 2.3] for the more general formulation of Proposition 35] and G, Section 2.3.2] for the formulation of the Division Algorithm.

Proposition 36. Let $J$ be the ideal generated by the elements

- $x_{i j} x_{j k}-x_{i k} x_{i j}-x_{j k} x_{i k}-\beta x_{i k}$, for $1 \leq i<j<k \leq n$,

- $x_{i j} y_{j k}-y_{i k} x_{i j}-y_{j k} y_{i k}-\beta y_{i k}$, for $1 \leq i<j<k \leq n$,

- $x_{i k} y_{j k}-y_{j k} y_{i j}-y_{i j} x_{i k}-\beta y_{i j}$, for $1 \leq i<j<k \leq n$,

- $x_{j k} y_{i k}-y_{i j} x_{j k}-y_{i k} y_{i j}-\beta y_{i j}$, for $1 \leq i<j<k \leq n$

in $R / Y$, where $Y$ is the ideal in $R$ generated by the elements

- $x_{i k} x_{i j} y_{i k}+x_{j k} x_{i k} y_{i k}+\beta x_{i k} y_{i k}-x_{i j} y_{i j} x_{j k}-x_{i j} y_{i k} y_{i j}-\beta x_{i j} y_{i j}$, for $1 \leq$ $i<j<k \leq n$.

Then there is a monomial order in which the above generators of $J$ form a Gröbner basis $\mathcal{G}$ of $J$ in $R / Y$, and the tips of the generators are, respectively,

$$
\begin{aligned}
& \text { - } x_{i j} x_{j k} \text {, } \\
& \text { - } x_{i j} y_{j k} \text {, } \\
& \text { - } x_{i k} y_{j k} \text {, } \\
& \text { - } x_{j k} y_{i k} \text {. }
\end{aligned}
$$

Proof. Let $x_{i j}>y_{k l}$ for any $i<j, k<l$, and let $x_{i j}>x_{k l}$ and $y_{i j}>y_{k l}$ if $(i, j)$ is less than $(k, l)$ lexicographically. The degree of a monomial is determined by setting the degrees of $x_{i j}, y_{i j}$ to be 1 and the degrees of $\beta$ and scalars to be 0 . A monomial with higher degree is greater in the order $>$, and the lexicographically greater monomial of the same degree is greater than the lexicographically smaller one. Since in $R$ two equal monomials can be written in two different ways due to commutations, we can pick a representative to work with, say the one which is the "largest" lexicographically among all possible ways of writing the monomial, to resolve any ambiguities. The order $>$ just defined is admissible; in it the tips of

- $x_{i j} x_{j k}-x_{i k} x_{i j}-x_{j k} x_{i k}-\beta x_{i k}$, for $1 \leq i<j<k \leq n$,

- $x_{i j} y_{j k}-y_{i k} x_{i j}-y_{j k} y_{i k}-\beta y_{i k}$, for $1 \leq i<j<k \leq n$,

- $x_{i k} y_{j k}-y_{j k} y_{i j}-y_{i j} x_{i k}-\beta y_{i j}$, for $1 \leq i<j<k \leq n$,

- $x_{j k} y_{i k}-y_{i j} x_{j k}-y_{i k} y_{i j}-\beta y_{i j}$, for $1 \leq i<j<k \leq n$

are

- $x_{i j} x_{j k}$,

- $x_{i j} y_{j k}$,

- $x_{i k} y_{j k}$

- $x_{j k} y_{i k}$.

In particular the generators of $J$ are tip reduced. A calculation of the overlap relations shows that $o\left(g_{1}, g_{2}, p, q\right) \Rightarrow_{\mathcal{G}} 0$ in $R / Y$, where $g_{1}, g_{2} \in \mathcal{G}$. Proposition 35 then implies Proposition 36.

Corollary 37. The reduced form of a good monomial $m$ is unique in $R / Y$.

Proof. Since the tips of elements of the Gröbner basis $\mathcal{G}$ of $J$ are exactly the monomials which we replace in the prescribed reduction rules $(5),(6),(7),\left(8^{\prime}\right)$, the reduced form of a good monomial $m$ is the remainder $r$ upon division by the elements of $\mathcal{G}$ with the order $>$ described in the proof of Proposition 36. Since we proved that in $R / Y$ the basis $\mathcal{G}$ is a Gröbner basis of $J$, it follows by [G. Proposition 2.7] that the remainder $r$ of the division of $m$ by $\mathcal{G}$ is unique in $R / Y$. That is, the reduced form of a good monomial $m$ is unique in $R / Y$. 
We would, however, like to prove uniqueness of the reduced form of a good monomial $m$ in $R$. This is what the next series of statements accomplishes.

Lemma 38. There is a monomial order in which the elements

- $x_{i k} x_{i j} y_{i k}+x_{j k} x_{i k} y_{i k}+\beta x_{i k} y_{i k}-x_{i j} y_{i j} x_{j k}-x_{i j} y_{i k} y_{i j}-\beta x_{i j} y_{i j}$, for $1 \leq$ $i<j<k \leq n$

are a Gröbner basis of $Y$ in $R$, and the tip of $x_{i k} x_{i j} y_{i k}+x_{j k} x_{i k} y_{i k}+\beta x_{i k} y_{i k}-$ $x_{i j} y_{i j} x_{j k}-x_{i j} y_{i k} y_{i j}-\beta x_{i j} y_{i j}$ is $x_{i j} y_{i k} y_{i j}$.

Proof. Let $x_{i j}<y_{k l}$ for any $i<j, k<l$, and let $x_{i j}>x_{k l}$ and $y_{i j}>y_{k l}$ if $(i, j)$ is less than $(k, l)$ lexicographically. The degree of a monomial is determined by setting the degrees of $x_{i j}, y_{i j}$ to be 1 and the degrees of $\beta$ and scalars to be 0 . A monomial with higher degree is greater in the order $>$, and the lexicographically greater monomial of the same degree, the variables being read from left to right, is greater than the lexicographically smaller one. Since in $R$ two equal monomials can be written in two different ways due to commutations, we can pick a representative to work with, say the one which is the "largest" lexicographically among all possible ways of writing the monomial, to resolve any ambiguities. The order $>$ just defined is admissible, the tip of $x_{i k} x_{i j} y_{i k}+x_{j k} x_{i k} y_{i k}+\beta x_{i k} y_{i k}-x_{i j} y_{i j} x_{j k}-x_{i j} y_{i k} y_{i j}-\beta x_{i j} y_{i j}$ is $x_{i j} y_{i k} y_{i j}$, and thus the generators of $Y$ are tip reduced. Since there are no overlap relations at all, by Proposition [35, Lemma 38 follows.

Corollary 39. If $f \in Y$, then there is a term of $f$ which can be written as $m_{1}$. $x_{i j} y_{i k} y_{i j} \cdot m_{2}$ for some $1 \leq i<j<k \leq n$, where $m_{1}, m_{2}$ are some monomials in $R$.

Proof. Lemma 38 implies that

$$
\left\langle x_{i j} y_{i k} y_{i j} \mid 1 \leq i<j<k \leq n\right\rangle=\langle\operatorname{Tip}(Y)\rangle .
$$

From here the statement follows.

Theorem 40. The reduced form of a good monomial $m$ is unique in $R$.

Proof. By Corollary 37, the reduced form of a good monomial $m$ is unique in $\mathbb{Q}\left\langle\beta, x_{i j}, y_{i j} \mid 1 \leq i<j \leq n\right\rangle / I_{\beta} / Y$. Since by Corollary 39 every $f \in Y$ contains a term divisible by $x_{i j} y_{i k} y_{i j}$ for some $1 \leq i<j<k \leq n$, it follows that the reduced form of a good monomial $m$ is unique in $\mathbb{Q}\left\langle\beta, x_{i j}, y_{i j} \mid 1 \leq i<j \leq n\right\rangle / I_{\beta}$, since a good monomial cannot contain any term divisible by $x_{i j} y_{i k} y_{i j}$ because of property (iii), with $\epsilon_{2}=+$.

A special case of Theorem 40 is the statement of Conjecture 2, since $w_{D_{n}}$ is a good monomial.

\section{ACKNOWLEDGEMENT}

The author is grateful to her advisor, Richard Stanley, for suggesting this problem and for many helpful suggestions. She would like to thank Alex Postnikov for sharing his insight into root polytopes and also Anatol Kirillov for his intriguing conjectures. 


\section{REFERENCES}

[BR] M. Beck, S. Robins, Computing the continuous discretely. Integer-point enumeration in polyhedra, Springer Science and Business Media, LLCC, 2007. MR2271992 (2007h:11119)

[D] E. Deutsch, Dyck path enumeration, Discrete Math. 204 (1999), 167-202. MR.1691869 (2000d:05007)

[FK] S. Fomin, A. N. Kirillov, Quadratic algebras, Dunkl elements and Schubert calculus, Advances in Geometry, Progress in Mathematics 172 (1999), 147-182. MR1667680 (2001a:05152)

[F] W. Fong, Triangulations and Combinatorial Properties of Convex Polytopes, Ph.D. Thesis, 2000.

[GGP] I. M. Gelfand, M. I. Graev, A. Postnikov, Combinatorics of hypergeometric functions associated with positive roots, in Arnold-Gelfand Mathematical Seminars: Geometry and Singularity Theory, Birkhäuser, Boston, 1996, 205-221. MR.1429893 (99k:33046)

[G] E. L. Green, Noncommutative Gröbner bases, and projective resolutions, Computational methods for representations of groups and algebras (Essen, 1997), 29-60, Progr. Math., 173, Birkhäuser, Basel, 1999. MR.1714602 (2001f:16030)

[K1] A. N. Kirillov, On some quadratic algebras, L. D. Faddeev's Seminar on Mathematical Physics, American Mathematical Society Translations: Series 2, 201, AMS, Providence, RI, 2000. MR 1772287 (2003a:05155)

[K2] A. N. Kirillov, personal communication, 2007.

[M] K. Mészáros, Root polytopes, triangulations, and the subdivision algebra, I, http:// arxiv.org/abs/0904.2194.

[P] A. Postnikov, Permutohedra, associahedra, and beyond, Int. Math. Res. Not. IMRN 2009, no. 6, 1026-1106. MR2487491 (2010g:05399)

[R1] V. Reiner, Quotients of Coxeter complexes and P-Partitions, Ph.D. Thesis, 1990.

[R2] V. Reiner, Signed posets, J. Combin. Theory Ser. A 62 (1993), 324-360. MR:1207741 (94d:06011)

[S1] R. Stanley, Decompositions of rational convex polytopes, Annals of Discrete Mathematics 6 (1980), 333-342. MR.593545 (82a:52007)

[S2] R. Stanley, Enumerative Combinatorics, vol. 2, Cambridge University Press, New York/Cambridge, 1999. MR1676282 (2000k:05026)

[Z1] T. Zaslavsky, Signed graphs, Discrete Appl. Math. 4 (1982), 47-74. MR676405 (84e:05095a)

[Z2] T. Zaslavsky, Orientation of signed graphs, Europ. J. Combinatorics 12 (1991), 361-375. MR 1120422(93a:05065)

Department of Mathematics, Massachusetts Institute of Technology, Cambridge, Massachusetts 02139 